\title{
Essential role for CCR6 in certain inflammatory diseases demonstrated using specific antagonist and knockin mice
}

\author{
Remy Robert, ${ }^{1}$ Caroline Ang, ${ }^{1}$ Cuizhi Sun, ${ }^{2}$ Laurent Juglair, ${ }^{1}$ Ee X. Lim, ${ }^{1}$ Linda J. Mason, ${ }^{1}$ \\ Natalie L. Payne, ${ }^{2}$ Claude C.A. Bernard, ${ }^{2}$ and Charles R. Mackay ${ }^{3}$ \\ 'Department of Biochemistry and Molecular Biology, Biomedicine Discovery Institute, Monash University, Clayton, \\ Australia. ${ }^{2}$ Australian Regenerative Medicine Institute, Monash University, Victoria, Australia. ${ }^{3}$ Department of \\ Microbiology, Biomedicine Discovery Institute, Monash University, Clayton, Australia.
}

\begin{abstract}
The chemokine receptor CCR6 marks subsets of T cells and innate lymphoid cells that produce IL-17 and IL-22, and as such may play a role in the recruitment of these cells to certain inflammatory sites. However, the precise role of CCR6 has been controversial, in part because no effective monoclonal antibody ( $m A b)$ inhibitors against this receptor exist for use in mouse models of inflammation. We circumvented this problem using transgenic mice expressing human CCR6 (hCCR6) under control of its native promoter (hCCR6-Tg/mCCR6 ${ }^{-/}$). We also developed a fully humanized mAb against hCCR6 with antagonistic activity. The expression pattern of hCCR6 in hCCR6-Tg/mCCR6-/- mice was consistent with the pattern observed in humans. In mouse models of experimental autoimmune encephalomyelitis (EAE) and psoriasis, treatment with anti-hCCR6 mAb was remarkably effective in both preventive and therapeutic regimens. For instance, in the imiquimod model of psoriasis, anti-CCR6 completely abolished all signs of inflammation. Moreover, anti-hCCR6 attenuated clinical symptoms of myelin oligodendrocyte glycoprotein-induced (MOG-induced) EAE and reduced infiltration of inflammatory cells in the central nervous system. CCR6 plays a critical role in Th17 type inflammatory reactions, and CCR6 inhibition may offer an alternative approach for the treatment of these lesions.
\end{abstract}

Conflict of interest: R. Robert and C.R. Mackay have received funding from Pfizer. C.R. Mackay has equity ownership of a biotechnology company, G2 Therapies (http://www.g2therapies. com.au), seeking to commercialize antiinflammatory therapies.

Submitted: May 2, 2017

Accepted: June 23, 2017

Published: August 3, 2017

\section{Reference information:}

JCI Insight. 2017;2(15):e94821.

https://doi.org/10.1172/jici.

insight.94821.

\section{Introduction}

New leukocyte subsets that drive chronic inflammation and autoimmune diseases have come to light in recent years. These include IL-17-producing Th17 cells (1), IL-22-producing Th22 cells (2, 3), as well as IL-17-producing innate cells including $\gamma \delta \mathrm{T}$ cells, invariant natural killer T cells (iNKTs) and group 3 innate lymphoid cells (ILC3). This diversity of leukocytes and related cytokines provides new opportunities for more targeted therapeutics that inhibit a narrower band of pathogenic cells or molecules. Blocking antibodies that interfere with the IL-23/IL-17 axis are an excellent example of this new knowledge translating to breakthrough medicines (4). Brodalumab (anti-IL-17 receptor A [anti-IL-17RA]), secukinumab (anti-IL-17A), and ustekinumab (anti-IL-12/23) show striking beneficial effects in human psoriasis (5). Nevertheless, effective treatment approaches are still lacking for many patients suffering from chronic immune-mediated inflammatory diseases, including psoriasis (anti-IL-17 nonresponders) or multiple sclerosis (MS), which appear to be driven by pathways in addition to IL-17.

Aside from targeting inflammatory cytokines, another promising strategy to inhibit excessive inflammation in tissues is to block recruitment of pathogenic immune cells from the blood $(6,7)$. Immune cell entry to tissues is orchestrated by both adhesion molecules and chemokines/chemokine receptors $(8,9)$. Interestingly, a hallmark of pathogenic IL-17-producing immune cells is that they all express high levels of the chemokine receptor CCR6 (10). Indeed, the transcription factor ROR $\gamma \mathrm{t}$ regulates both Th17 differentiation and CCR6 expression (11). Furthermore, the production of CCL20 (MIP3 $\alpha$ ), the main ligand for CCR6, is dramatically increased in numerous inflammatory and/or autoimmune conditions and facilitates migration of IL-17-producing cells to inflamed sites (12-14). Various studies using CCR6-deficient mice illustrate the importance of this axis in autoimmune and inflammatory disorders (15-18). 
Antagonizing CCR6 with a monoclonal antibody $(\mathrm{mAb})$ should be an effective strategy for the treatment of Th17- or Th22-mediated inflammatory and autoimmune diseases. This provides an exciting opportunity to selectively inhibit distinct arms of the T cell response, particularly in diseases where IL-17 and/ or IL-22 play a major role. In fact, CCR6 $6^{+}$Th17-expressing cells may express a number of inflammatory cytokines, including IL-21 and to a lesser extent IFN- $\gamma$ that are involved in the propagation of inflammatory immune responses (19).

In the present study, we have isolated and humanized a mAb specific for human CCR6 (hCCR6) named $6 \mathrm{H} 12$. In order to avoid Fc-mediated effector functions, a human heavy chain (hIgG1) with greatly reduced antibody-dependent cell-mediated cytotoxicity (ADCC) was used. The humanized 6H12 (h6H12) was produced in $\mathrm{CHO}$ cells and showed binding and neutralizing behavior that was similar to that of the parental mouse $6 \mathrm{H} 12 \mathrm{IgG}(\mathrm{m} 6 \mathrm{H} 12)$. We showed that our antibody binds to hCCR6 with high affinity and can block CCL20-mediated chemotaxis. h6H12 did not cross-react with mouse CCR6 and was therefore unsuitable for mouse studies, and no effective surrogate mAbs against mouse CCR6 (mCCR6) have been produced, so we developed hCCR6-Tg/mCCR6 ${ }^{-/-}$mice for preclinical studies. In 2 models of chronic inflammation (experimental autoimmune encephalomyelitis [EAE] and imiquimod-induced [IMQ-induced] skin inflammation), our humanized $\mathrm{mAb}$ showed remarkable inhibition of disease in hCCR6-Tg/mCCR6 ${ }^{-1-}$ mice that greatly exceeded that seen with anti-IL-17 mAb.

\section{Results}

Generation of potentially novel anti-hCCR6 $\mathrm{mAbs}$. We sought to generate mAbs against CCR6 to inhibit the function of this receptor and to evaluate the effect of antagonizing CCR6 in certain inflammatory reactions in vivo. mAbs against hCCR6 were generated by immunizing C57BL/ 6 mice with the murine pre-B cell lymphoma line, L1.2, which expresses high levels of transfected hCCR6. Dozens of clones were initially isolated. We focused our efforts on $2 \mathrm{mAbs}$, termed 6H12 and 29A6, that reacted with CCR6-transfected L1.2 cells, but not with L1.2 cells expressing other well-validated chemokine receptor transfectants (Figure 1, A and B). Moreover, 6H12 and 29A6 showed an identical pattern of reactivity against human leukocytes, as previously noted for various commercially available anti-CCR6 antibodies (Figure 1C and Supplemental Figure 1; supplemental material available online with this article; https://doi.org/10.1172/jci. insight.94821DS1). In particular, they stained most B cells (data not shown) as well as $\mathrm{T}$ cell subsets in the $\mathrm{CD}^{+} / \mathrm{CD}^{+}$and $\mathrm{CD}^{+} / \mathrm{CD}^{+}$compartments (Figure $1 \mathrm{C}$ ).

To determine the fine specificity of the mAbs, they were first screened by ELISA against peptides corresponding to the N-terminal 1 (aa 1-28), N-terminal 2 (aa 18-46), extracellular loop 1 (ECL1), ECL2, and ECL3 regions of CCR6. All mAbs reacted with the peptide corresponding to aa $1-28$ of the N-terminal region, except $1 \mathrm{mAb}$ (29A6), which recognized peptide 18-46 (data not shown). Knowing that all the $\mathrm{mAbs}$ were directed against the $\mathrm{N}$-terminal region of CCR6, epitope refinement experiments were next performed by ELISA using overlapping 12-mer peptides covering the sequence 1-40 (1-mer overlap). Interestingly, 29A6 and 6H12 recognize nonoverlapping epitopes (Figure 1E). These results were confirmed in a competitive flow cytometry experiment where $6 \mathrm{H} 12$ at high concentration was unable to inhibit binding of 29A6 towards human B cells (Figure 1D). This enabled mAb 29A6 to be used as a detection mAb in vivo, in the presence of $6 \mathrm{H} 12$.

$6 H 12$ mAb engineering. As a first step in the antibody engineering process, $6 \mathrm{H} 12$ was humanized by complementarity-determining region (CDR) grafting. The VH and VL sequences of the m6H12 mAb were compared with the functional human germline $\mathrm{V}$ and $\mathrm{J}$ gene repertoires using IMGT/V-QUEST and IMGT/Junctions analysis tools. In the case of the heavy chain, the human germline $\mathrm{V}$ and $\mathrm{J}$ genes, IGHV2-5*08 and IGHJ $4 * 01$, exhibited the highest homology with their murine counterparts, sharing $79 \%$ and $85 \%$ identity, respectively. For the light chain, human IGKV2-28*01 and IGKJ4*02 genes displayed the highest homologies ( $80 \%$ and $81 \%$, respectively) with their murine equivalent sequences. These human genes were selected as acceptor sequences for the grafting of the murine CDRs while taking care to leave the mouse residues in the Vernier zone untouched. The h6H12 mAb genes were further cloned in our in-house hIgG1 vector for expression in $\mathrm{CHO}$ cells, and the mAb was purified from supernatant by affinity chromatography on a protein A column. The h6H12 binding characteristics were then compared to the parental m6H12 mAb by flow cytometry on hCCR6 L1.2 cells. The EC50 values demonstrated that the engineered $\mathrm{mAb}$ retained full affinity towards hCCR6, with both antibodies showing binding in the $\mathrm{pM}$ range (Figure $1 \mathrm{~F}$ ). 
A

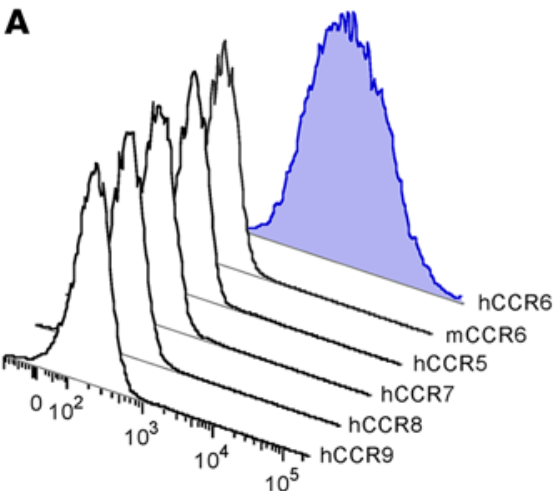

B
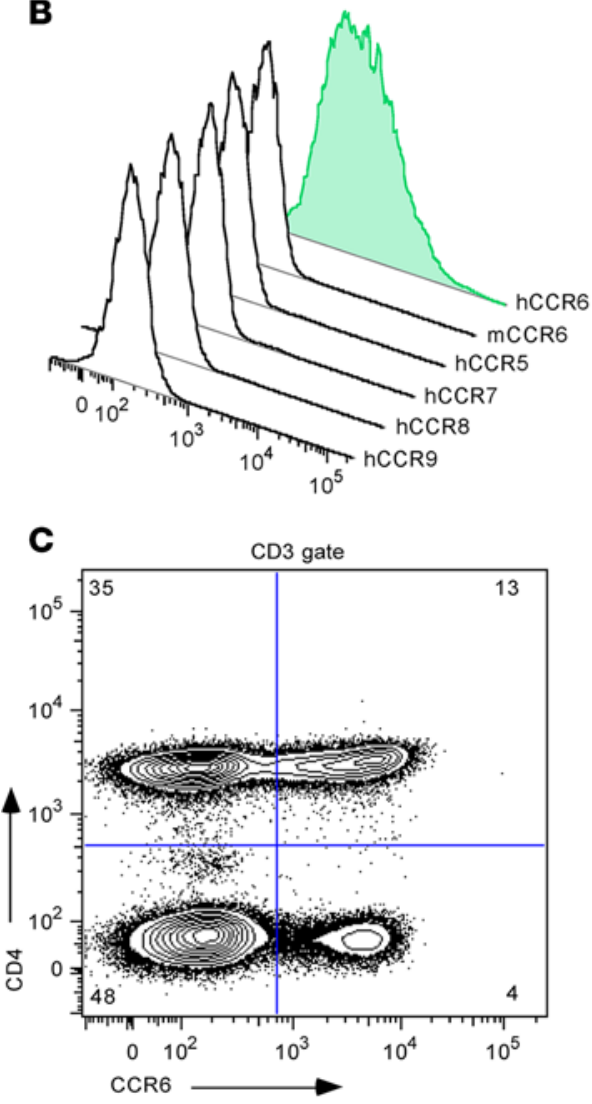

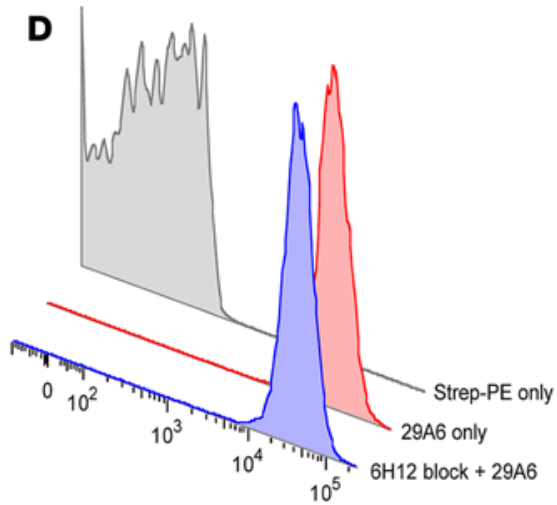

$\mathbf{E}$

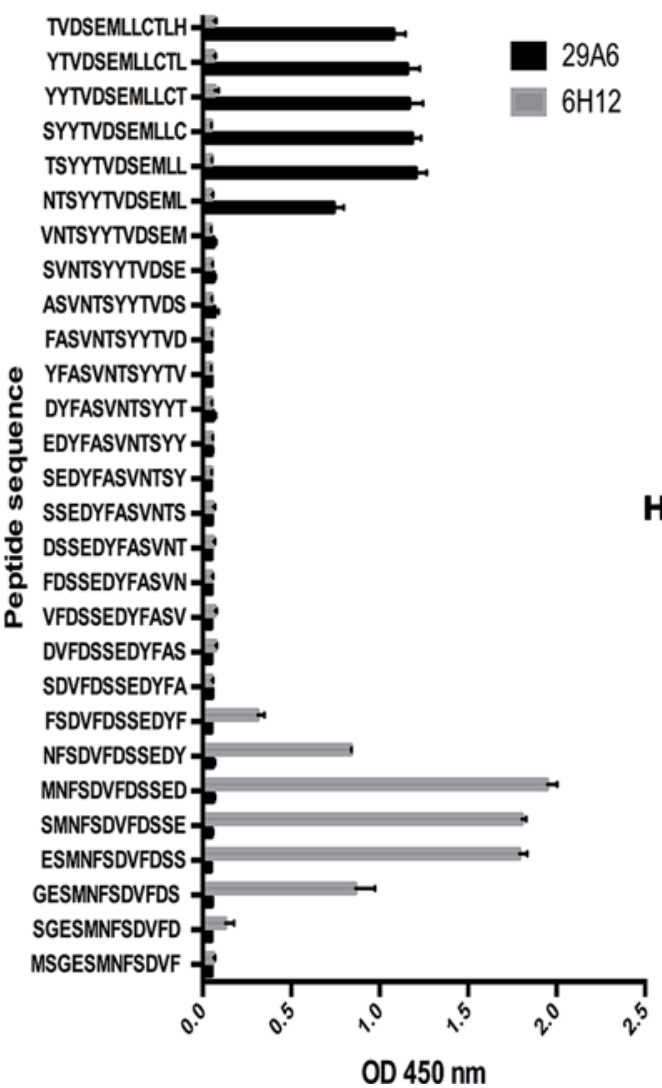

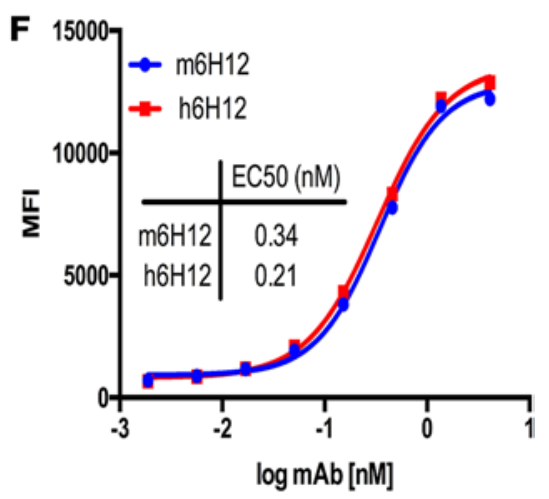

G
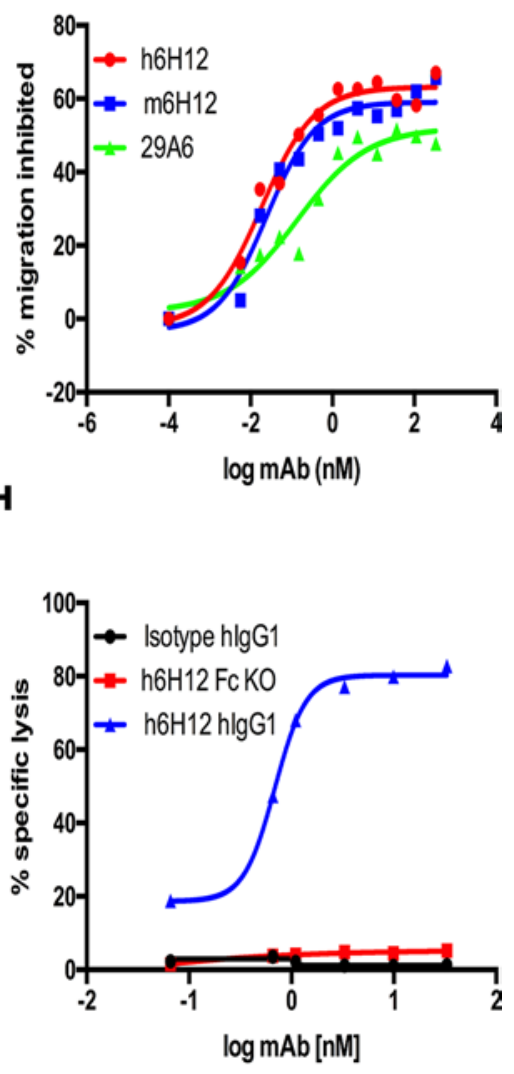

Figure 1. Characterization and engineering of anti-CCR6 mAb. mAbs 6H12 (A) and $29 A 6$ (B) staining of various L1.2 transfectants. Stable L1.2 transfectants expressing either human CCR6 (hCCR6), mouse CCR6 (mCCR6), hCCR5, hCCR7, hCCR8, or hCCR9 were stained with the anti-hCCR6 mAbs 6H12 and 29A6. (C) A 3-color staining protocol was used to assess for expression of CCR6 (horizontal axis) on human T cells (CD3 ${ }^{+} / \mathrm{CD}^{+}$) using $6 \mathrm{H} 12$ FITC-conjugated mAb. (D) Binding of both $6 \mathrm{H} 12$ and $29 \mathrm{~A} 6 \mathrm{mAbs}$ to human CD19+ cells was determined by preincubating cells with $30 \mu \mathrm{g} / \mathrm{ml}$ purified $6 \mathrm{H} 12$ (block) followed by staining with $1 \mu \mathrm{g} / \mathrm{ml} 29 \mathrm{A6}-\mathrm{FITC}$. (E) Mapping of $6 \mathrm{H} 12$ (gray bars) and $29 \mathrm{A6}$ (black bars) binding sites on human CCR6 $\mathrm{N}$-terminal region by peptide ELISA. (F) Comparison of humanized $6 \mathrm{H} 12$ (h6H12) versus mouse $6 \mathrm{H} 12$ (m6H12) by flow cytometry. EC50 of h6H12 and m6H12 was measured by flow cytometry on hCCR6 L1.2 cells using increasing concentrations of purified mAbs. (C) Inhibition of hCCL20-mediated chemotaxis by anti-hCCR6 mAbs. Assessment of hCCR6 L1.2 cell chemotactic response to $120 \mathrm{nM} \mathrm{CCL20} \mathrm{was} \mathrm{carried} \mathrm{out} \mathrm{in} \mathrm{the} \mathrm{presence} \mathrm{of} \mathrm{increasing}$ concentrations of purified anti-CCR6 mAbs. The percentage of total chemotaxis was calculated using the number of cells migrated in the absence of mAb as 100\%. (H) Cytotoxicity of activated human NK cells (effector) against hCCR6 L1.2 cells (target) by mAbs in vitro. Dose-response relationship was obtained using increasing amounts of h6H12 IgG1 (blue curve), h6H12 Fc-KO (red curve), or hlgG1 isotype control (black curve) and a constant effector/target cell ratio of 2:1. 
A
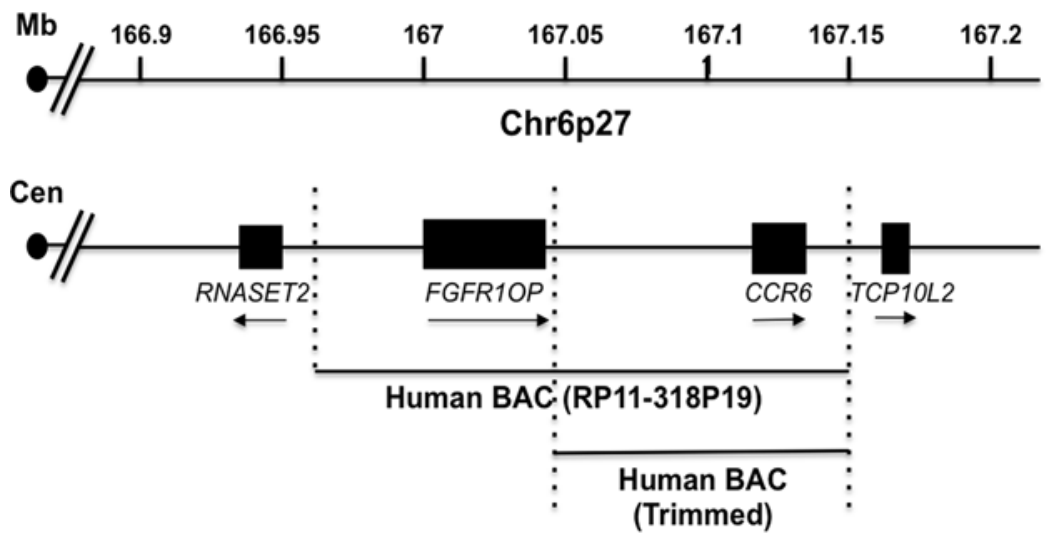

C
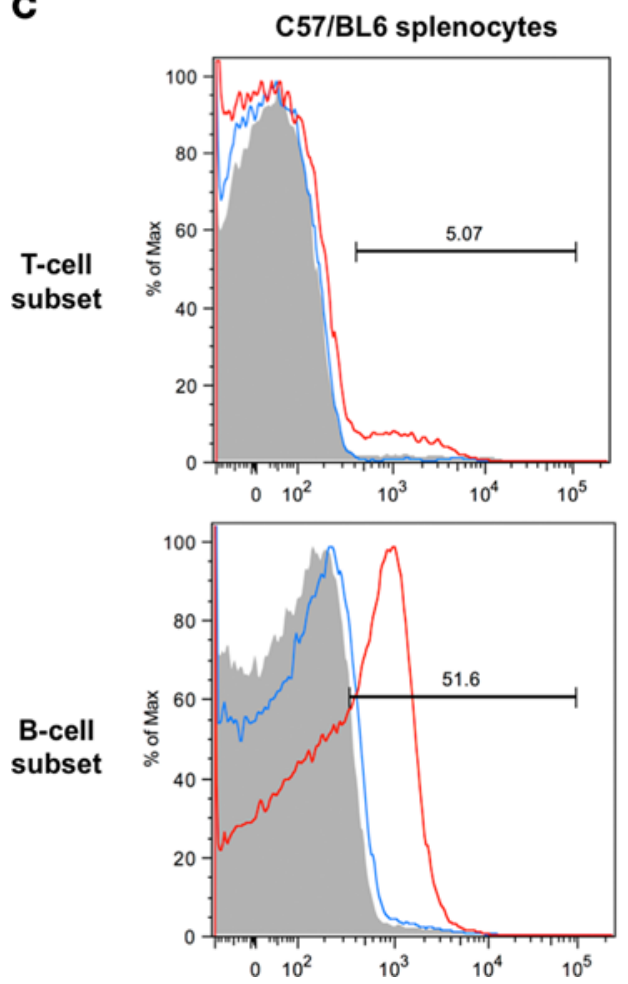

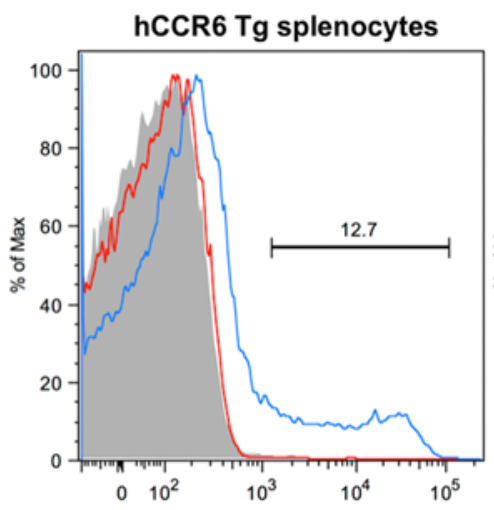

B
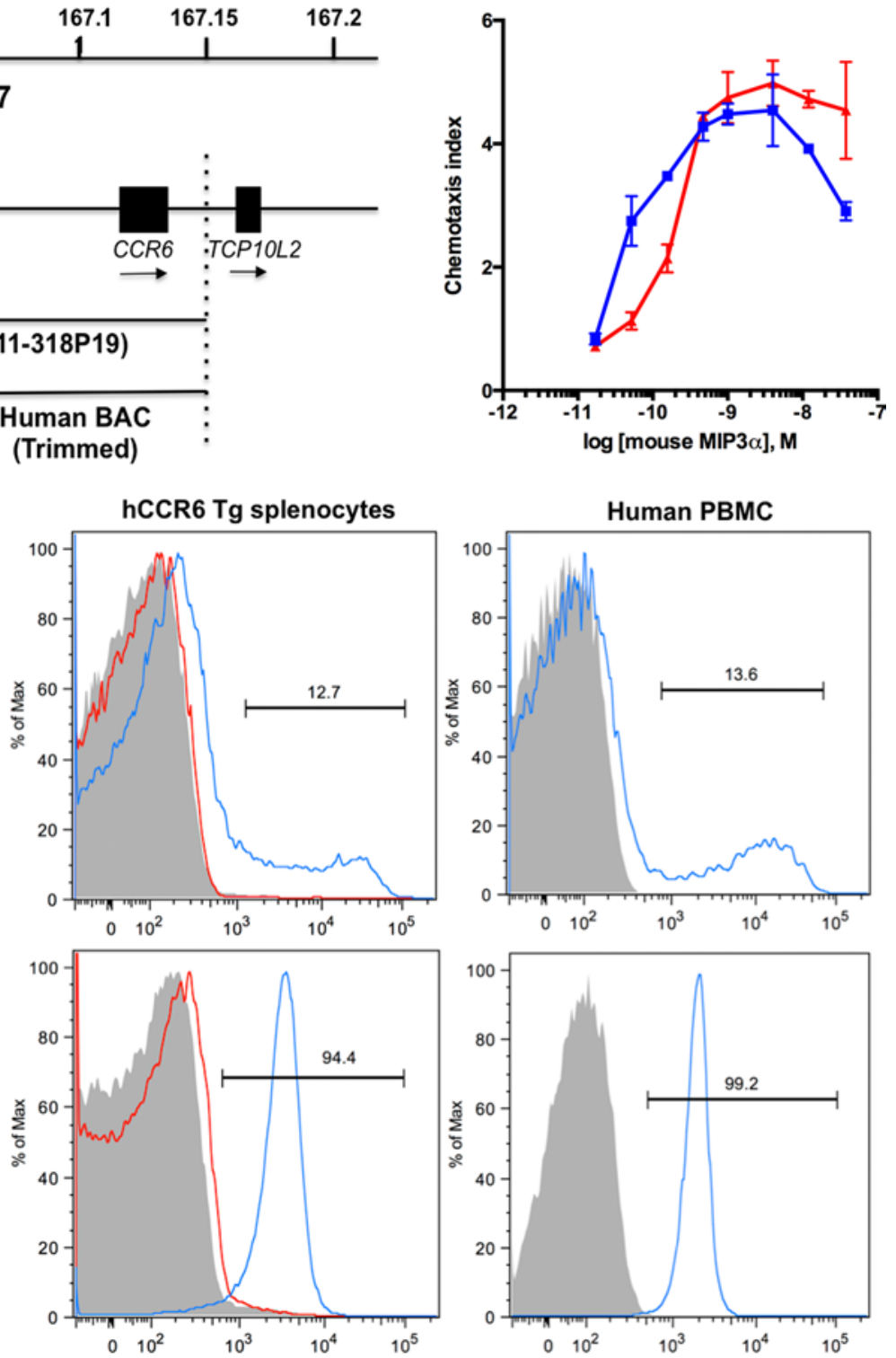

Figure 2. Characterization of hCCR6-Tg/mCCR6-/- mice. (A) hCCR6-Tg/mCCR6-1- mice were constructed using a bacterial artificial chromosome (BAC) encompassing the human CCR6 locus. (B) Purified CD3 ${ }^{+}$T cells from hCCR6-Tg/mCCR6 ${ }^{-1-}$ mice (blue line) or WT mice (red line) show similar chemotactic activity towards the mouse CCL20 ligand. (C) The expression of mCCR6 (clone FAB590A, red line) or hCCR6 (clone G034E3, blue line) on T cells (top panels) or B cells (bottom panels) was compared between WT (left panels), hCCR6-Tg/mCCR6-1- (middle panels), and human peripheral blood mononuclear cells (PBMCs) (right panels). The gray histograms represent the isotype controls.

Secondly, we introduced mutations in the hIgG1 Fc region (20) to produce a fully humanized antibody devoid of effector functions (h6H12 Fc-KO). We tested mAb h6H12 Fc-KO for its ability to induce ADCC in vitro. As shown in Figure 1H, h6H12 WT hIgG1 induced cell death in a dose-dependent manner when incubated with target (hCCR6 L1.2) and effector cells (human NK). In contrast, even at high concentrations, $\mathrm{h} 6 \mathrm{H} 12 \mathrm{Fc}-\mathrm{KO} \mathrm{mAb}$ was unable to induce cell death.

Inhibition of chemotaxis to CCL20 using anti-CCR6 mAbs. To evaluate the ability of our mAbs to inhibit CCL20mediated migration, chemotaxis experiments were performed. hCCR6 L1.2-transfected cells were allowed to migrate in transwell plates towards optimal concentrations of recombinant CCL20 $\left(\mathrm{EC}_{90}=120 \mathrm{nM}\right)$. All mAbs substantially reduced cell migration in a dose-dependent manner (Figure 1G). These results also highlight the importance of the CCR6 N-terminal region in CCL20 binding and signaling, as previously described with chimeric hCCR6 constructs (21). Among the mAbs, h6H12 Fc-KO showed the best inhibition profile. 
Generation and characterization of $h C C R 6-\mathrm{Tg} / \mathrm{mCCR} 6^{-1-}$ mice. In addition to the anti-human mAb generation efforts described above, we sought to generate anti-mCCR $6 \mathrm{mAbs}$. For this, we used $\mathrm{Crr}^{-1-}$ mice in an effort to circumvent tolerance to mCCR6. Despite approximately 4 fusions and the screening of over 8,000 hybridomas, not a single anti-mCCR $6 \mathrm{mAb}$ was generated. Therefore, we generated hCCR6-Tg/mCCR6 ${ }^{-1}$ mice using the complete genomic hCCR6 sequence isolated from a BAC clone (Figure 2A). Excised DNA fragments of $120 \mathrm{kbp}$ were microinjected into the pronuclei of fertilized eggs from C57BL/6 mice. Blood from founder mice was immunophenotyped using a specific anti-hCCR6 mAb (clone G034E3). mCCR6-KO mice (C57BL/6 background) (22) were backcrossed with the founder showing the highest hCCR6 expression and the progenies were inbred for more than 10 generations to produce hCCR6-Tg/ mCCR $6^{-1-}$ mice. We postulated that the regulatory sequences included in the DNA fragment might result in a cell-specific expression in mice similar to that in humans. To test this, we compared by flow cytometry the expression pattern of CCR6 in lymphocyte subsets from WT C57BL/6 mice using a commercial anti-mCCR6 mAb (clone FAB590A), and hCCR6-Tg/mCCR6 ${ }^{-1-}$ mice and human peripheral blood mononuclear cells (PBMCs) using a commercial anti-hCCR6 mAb (Figure 2C). Interestingly, CCR6 expression in WT C57BL/6 mice and human lymphocytes was different, since all human B cells expressed CCR6, compared with approximately $55 \%$ of B cells in mice. A larger proportion of human $\mathrm{CD}^{+}$cells expressed the receptor $(\sim 14 \%)$ compared with mouse $\mathrm{CD}^{+}$cells $(\sim 5 \%)$. Thus, the introduction of human regulatory regions in hCCR6-Tg/mCCR6 ${ }^{-1-}$ mice gave rise to a CCR6 expression pattern resembling that observed in human PBMCs, rather than in WT C57BL/6 mouse splenocytes. We conclude that regulatory regions of the hCCR6 gene may account for species differences in levels of expression. However, it is also conceivable that the difficulty in generating quality anti-mCCR6 mAbs resulted in the one $\mathrm{mAb}$ that has been produced to date yielding suboptimal staining.

For meaningful in vivo analyses, the mouse ligand for CCR6, CCL20, should bind and function with hCCR6, similar to its binding to mCCR6. We assessed the ability of mouse CCL20 (mCCL20) to bind and induce migration of $\mathrm{CD}^{+}$cells isolated from hCCR6-Tg/mCCR6 ${ }^{-1-}$ mice. As shown in Figure $2 \mathrm{~B}$, mCCL20 induced chemotaxis of hCCR6 T cells as efficiently as that for T cells isolated from WT C57BL/6 mice. Thus, the expression and function of hCCR6 in our transgenic line was as we expected and provided a tool to assess CCR6 function in vivo, in inflammatory models.

Anti-CCR6 treatment prevents and inhibits clinical progression in established EAE. Previous studies indicated that CCR6/CCL20 interaction was crucial for the development of EAE, since CCR6 ${ }^{-1-}$ mice were resistant to EAE (15) and anti-CCR6 polyclonal antibody treatment prevented the progression of disease (23). Murine EAE was induced by injection of recombinant mouse myelin oligodendrocyte glycoprotein (rmMOG), a model in which antigen-specific B cells contribute to disease pathogenesis (24). Importantly, hCCR6-Tg/mCCR6 ${ }^{-1-}$ mice develop EAE in a manner that is similar to that of WT C57BL/ 6 mice (Supplemental Figure 2). Moreover, following immunization with rmMOG, a significant increase in hCCR6 expression was observed on $\mathrm{CD}^{+}$and $\mathrm{CD} 8^{+} \mathrm{T}$ cells isolated from lymph nodes and spleen (Supplemental Figure 2).

To determine whether CCR6 inhibition with humanized mAb h6H12 Fc-KO could alter the clinical severity of the disease in hCCR6-Tg/mCCR6 ${ }^{-1-}$ mice, we administered mAb h6H12 Fc-KO i.p. on day 8 and 13 after immunization and compared the effect with an anti-mouse IL-17 mAb (clone 17F3) (25-27). Remarkably, injection of our anti-CCR6 $\mathrm{mAb}$ at $5 \mathrm{mg} / \mathrm{kg}$ significantly reduced disease severity, in comparison with an isotype control (Figure 3A). The mean cumulative (Figure 3C) and maximal clinical scores (Figure 3D) were also decreased. Likewise, h6H12 mAb treatment markedly increased the number of disease-free animals (Figure 3B). The analysis of spinal cord sections demonstrated that h6H12 Fc-KOtreated mice had fewer inflammatory leukocytes infiltrating, with a concomitant preservation of the myelin content and notably less axonal damage compared with anti-IL-17- and isotype control mAb-treated animals (Figure 3, E and F).

Bolstered by the prophylactic regimen data, we performed a more clinically relevant evaluation of the therapeutic potential of our $\mathrm{mAb}$ in hCCR6-Tg/mCCR6 ${ }^{-/-}$mice that were already exhibiting symptoms of EAE. rmMOG-induced mice with EAE scores of 1.0 were treated with either h6H12 Fc-KO mAb or an isotype control antibody. After a 2-week treatment, the anti-hCCR6 mAb group showed significantly lower EAE scores than the control group. Most notably, disease progression appeared stabilized (Figure 3G).

At 24 days after immunization, CNS tissue was collected and infiltrating CD4 ${ }^{+} \mathrm{T}$ cells were analyzed by flow cytometry (Figure 4, A and B). We observed a paucity of CD45 $5^{\text {hi }}$ cells in the anti-IL-17- and 

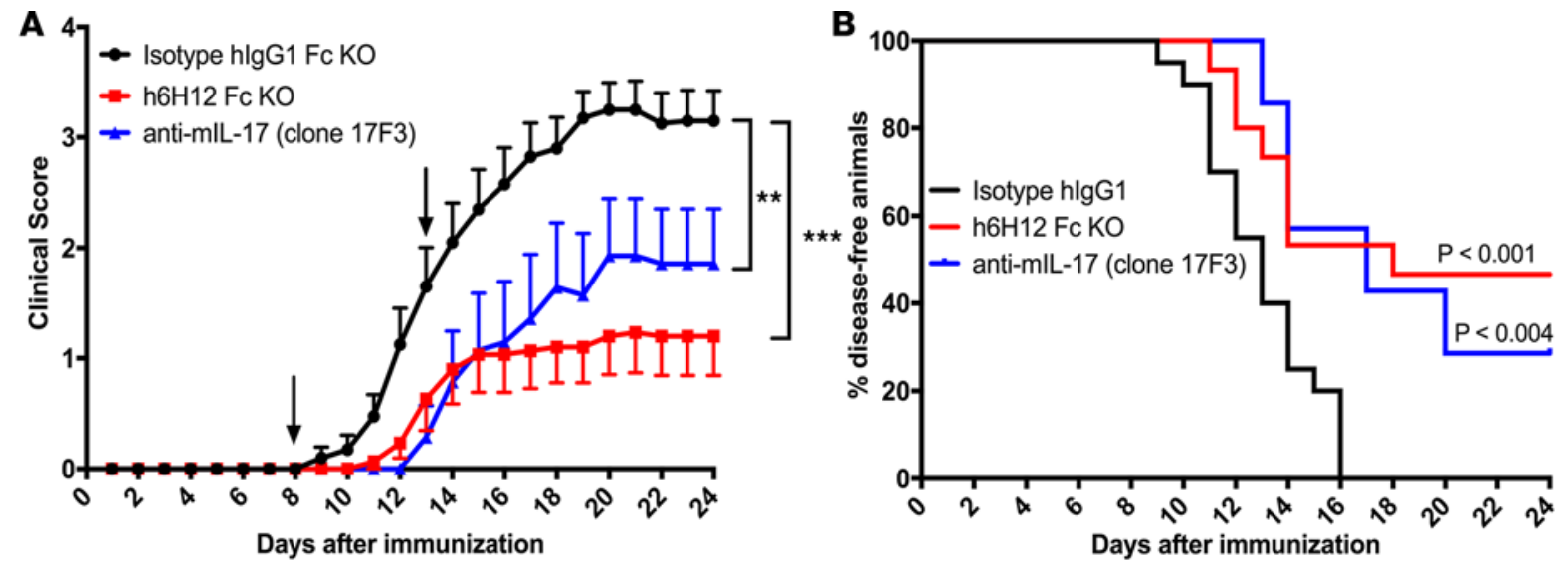

C
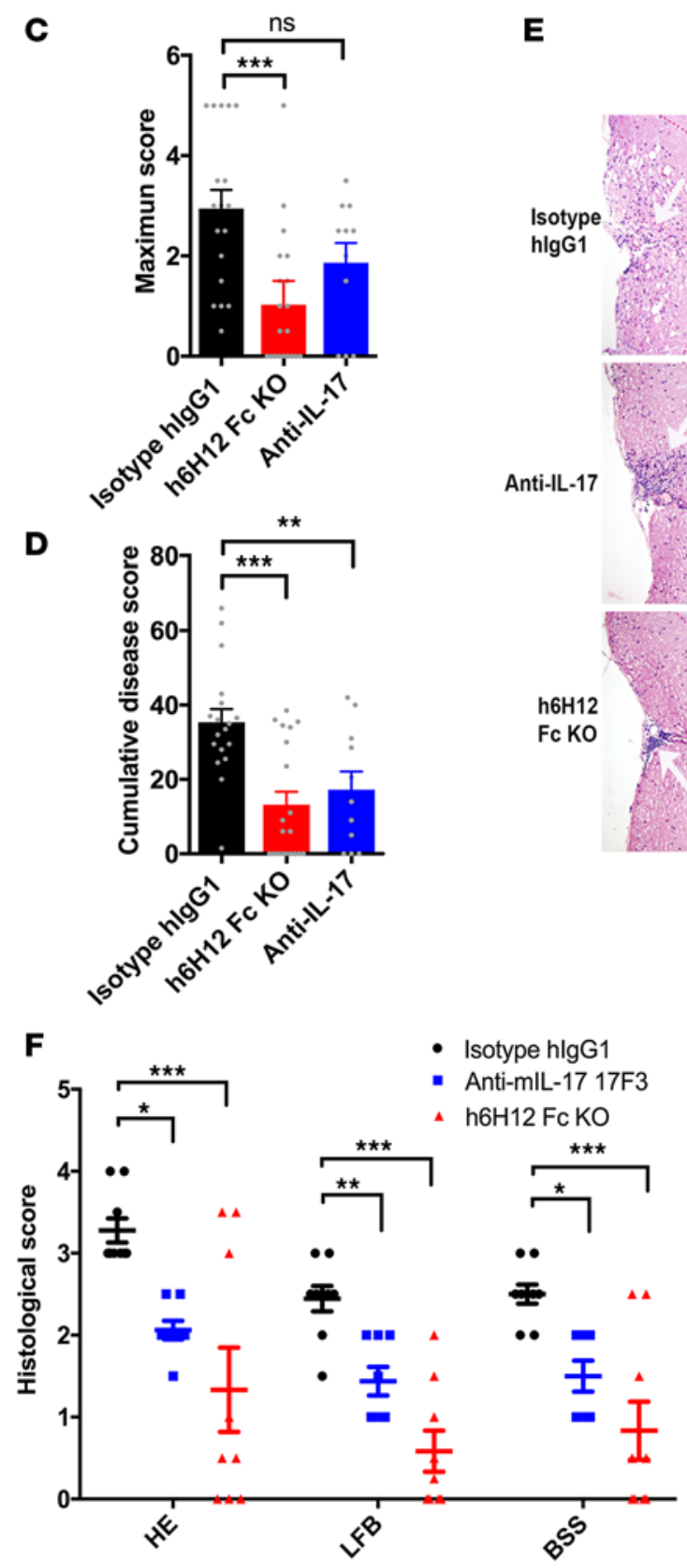

E

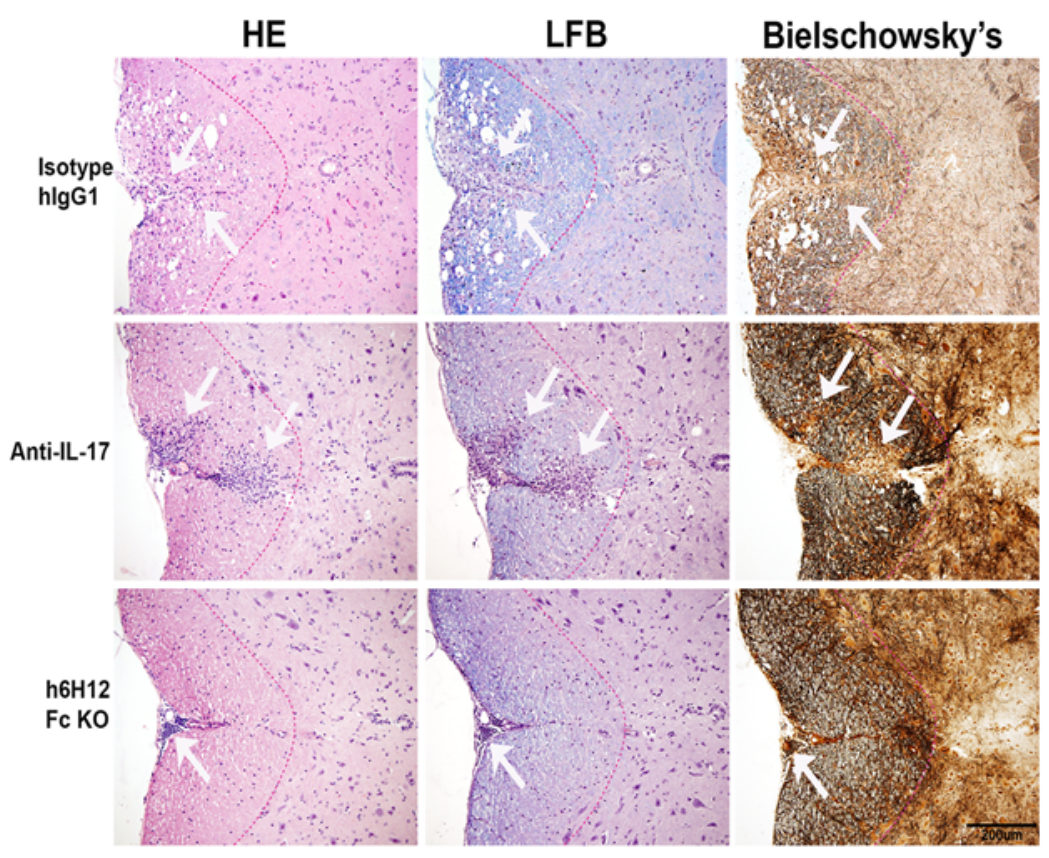

G

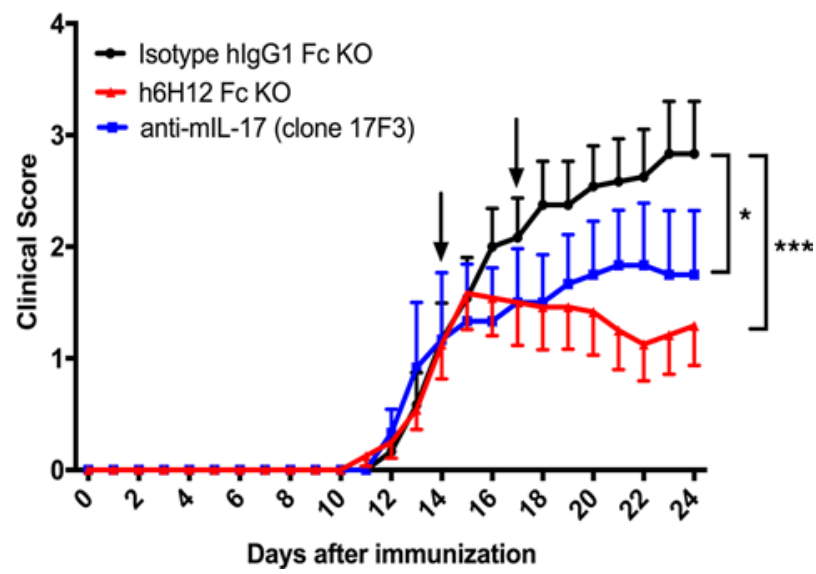

Days after immunization 
Figure 3. Anti-hCCR6 mAb prevents and inhibits EAE in hCCR6-Tg/mCCR6-- mice. (A) Experimental autoimmune encephalomyelitis (EAE) preventive study. Blockade of human CCR6 (hCCR6) with humanized 6H12 (h6H12) mAb (2 mg/kg, black arrows) prevents the development of EAE in hCCR6-Tg/ mCCR6 ${ }^{-1}$ mice immunized with recombinant mouse (rm) myelin oligodendrocyte glycoprotein (MOC). (B) Kaplan-Meier plot of the proportion of diseasefree animals after EAE induction in isotype ( $n=18$, black line) versus h6H12 Fc-KO-treated ( $n=20$, red line) and anti-mlL-17 mAb-treated animals ( $n$ $=11$, blue line) (Mantel-Cox log-rank test). (D) Maximum and (C) cumulative disease scores in isotype- $(n=18)$, h6H12 Fc-KO- $(n=20)$, and anti-mlL-17 mAb-treated mice $(n=11)$. (E) Representative stained histological sections of spinal cords from immunized animals treated with isotype control (top panels), h6H12 Fc-KO (middle panels), or anti-mIL-17 (bottom panels). Serial sections were stained with H\&E to determine the degree of inflammatory cell infiltrates, luxol fast blue (LFB) to establish myelin integrity, and Bielschowsky silver stain (BSS) to ascertain axonal loss and damage (white arrows indicate area of leukocyte infiltration). Scale bar: $200 \mu \mathrm{m}$. (F) Histological scores for inflammation (H\&E), demyelination (LFB), and axonal damage (BSS) (blacks bars, isotype control [ $n=9$ ]; blue bars, anti-mIL-17 [ $n=8]$; red bars, h6H12 Fc-KO $[n=9])$. (G) EAE therapeutic study. Injections of h6H12 (5 mg/kg; black arrow) significantly reduce the disease development ( $n=12$, red curve) compared with mice treated with an isotype control mAb ( $n=12$, black curve) or anti-mIL-17 mAb ( $n=8$, blue curve). The clinical course of EAE (A and $\mathbf{G}$ ) was analyzed with the nonparametric Kruskal-Wallis ANOVA with the Dunn's post-hoc test. Histological, maximum clinical, and cumulative scores (C, D, and F) were compared by 1-way ANOVA with Dunnett's multiple comparisons test relative to the control isotype-treated group. ${ }^{*} P<0.05,{ }^{* *} P<0.01,{ }^{* * *} P<0.001$.

h6H12-treated groups compared with the isotype control group (Figure 4, A and B; first row). Among the $\mathrm{CD} 4^{+}$cells, there was a marked reduction in both the frequency and total number of CCR $6^{+}$cells in the anti-IL-17- and h6H12-treated mice compared with the mice that received the isotype control (Figure 4, A and B; second row). Intracellular cytokine staining on $\mathrm{CD}^{+}{ }^{+} \mathrm{T}$ cells further revealed that IL-17 expression was significantly higher in the isotype-treated group (Figure 4, A and B; third row). Notably, both anti-IL-17- and anti-hCCR6 $\mathrm{mAb}$-treated groups showed a reduction of infiltrating CCR6 ${ }^{+}$Tregs compared with the isotype-treated group (Figure 4, A and B; fourth row). Interestingly, a proportion of Th1 cells $\left(\mathrm{CD}^{+} / \mathrm{CD}^{+} / \mathrm{IFN}-\gamma^{+}\right)$also express $\mathrm{CCR}^{+}$(Figure 4 , A and B; fifth row) and their number was significantly lower in the anti-IL-17 and h6H12 mAb groups compared with the isotype-treated group. Thus, anti-CCR6 treatment suppressed the infiltration of pathogenic Th17 cells into the CNS.

Anti-CCR6 treatment prevents IMQ-induced skin inflammation and inhibits clinical progression in established disease. The importance of the IL-23/Th17 axis, and in particular CCR6 and CCL20, in psoriasis has been established both in animal models $(16,28)$ and in human disease (29). Indeed, CCR6 and CCL20 are expressed at significantly higher levels in human psoriatic skin than in normal skin, and CCR6 expression is higher on circulating PBMCs from psoriasis patients compared with normal donors (30).

Psoriasiform skin inflammation induced by IMQ in hCCR6-Tg/mCCR6 $6^{-/}$mice was used to investigate the therapeutic potential of our mAb. Notably, C57BL/6 and hCCR6-Tg/mCCR6 $6^{-1-}$ mice showed similar disease kinetics when IMQ cream was applied topically on shaved backs (Supplemental Figure 2). We therefore first sought to investigate the prophylactic effects of CCR6 inhibition by injecting h6H12 Fc-KO mAb and to compare this to anti-IL-17 immunotherapy. hCCR6-Tg/mCCR6-/- mice treated with IMQ and injected with the isotype control $\mathrm{mAb}$ displayed a typical increase in skin thickness and scale formation (Figure 5, A and B). Strikingly, mAb h6H12 Fc-KO-treated mice failed to show any visible scales or skin thickening (Figure 5, A and B) and the therapeutic effects appeared superior to that observed with the anti-IL-17 treatment, at the same dose regimen. Histological analysis confirmed these clinical observations, as the skin from h6H12 Fc-KO-treated mice showed reduced epidermal acanthosis, hyperkeratosis, and leukocyte infiltration compared with isotype control-injected mice, and importantly also with antiIL-17-injected animals (Figure 5A).

In order to address the therapeutic potential of our mAb, mice were treated with IMQ until a $30 \%$ increase in back skin thickness was reached. The isotype control, 17F3, and h6H12 Fc-KO mAbs were then injected every 2 days and the mice were monitored daily for 7 days. As shown in Figure 5C, anti-hCCR6 significantly reduced the rate of skin thickening in this therapeutic regimen.

Recent studies established that the main source of IL-17 in IMQ- and IL-23-induced psoriasis in mice was not Th17 CD $4^{+}$cells, but a distinct population of $\gamma \delta^{10} C C R 6^{\text {hi }}$ cells $(16,31,32)$. Importantly, humans appear to carry an equivalent dermal $\gamma \delta \mathrm{T}$ cell population, likewise prone to secrete IL-17 and also implicated as playing a pathogenic role in psoriasis (32). Therefore, we analyzed by flow cytometry single-cell suspensions derived from the skin of mice treated either with the Vaseline control cream, IMQ plus isotype control mAb, IMQ plus anti-mIL-17 mAb, or IMQ plus anti-hCCR6 mAb. As shown in Figure $5 \mathrm{~A}, \mathrm{mAb}$ h6H12 $\mathrm{Fc}-\mathrm{KO}$ and anti-IL-17 mAb treatment dramatically reduced the number and frequency of pathogenic $\gamma \delta^{\mathrm{lo}} \mathrm{CCR} 6^{\mathrm{hi}}$ cells compared with isotype control-treated mice (Figure 6B; first row). These results are consistent with those obtained by Mabuchi et al., who demonstrated that anti-mCCL20 treatment in an IL-23-induced skin inflammation model reduced psoriasiform dermatitis 
A
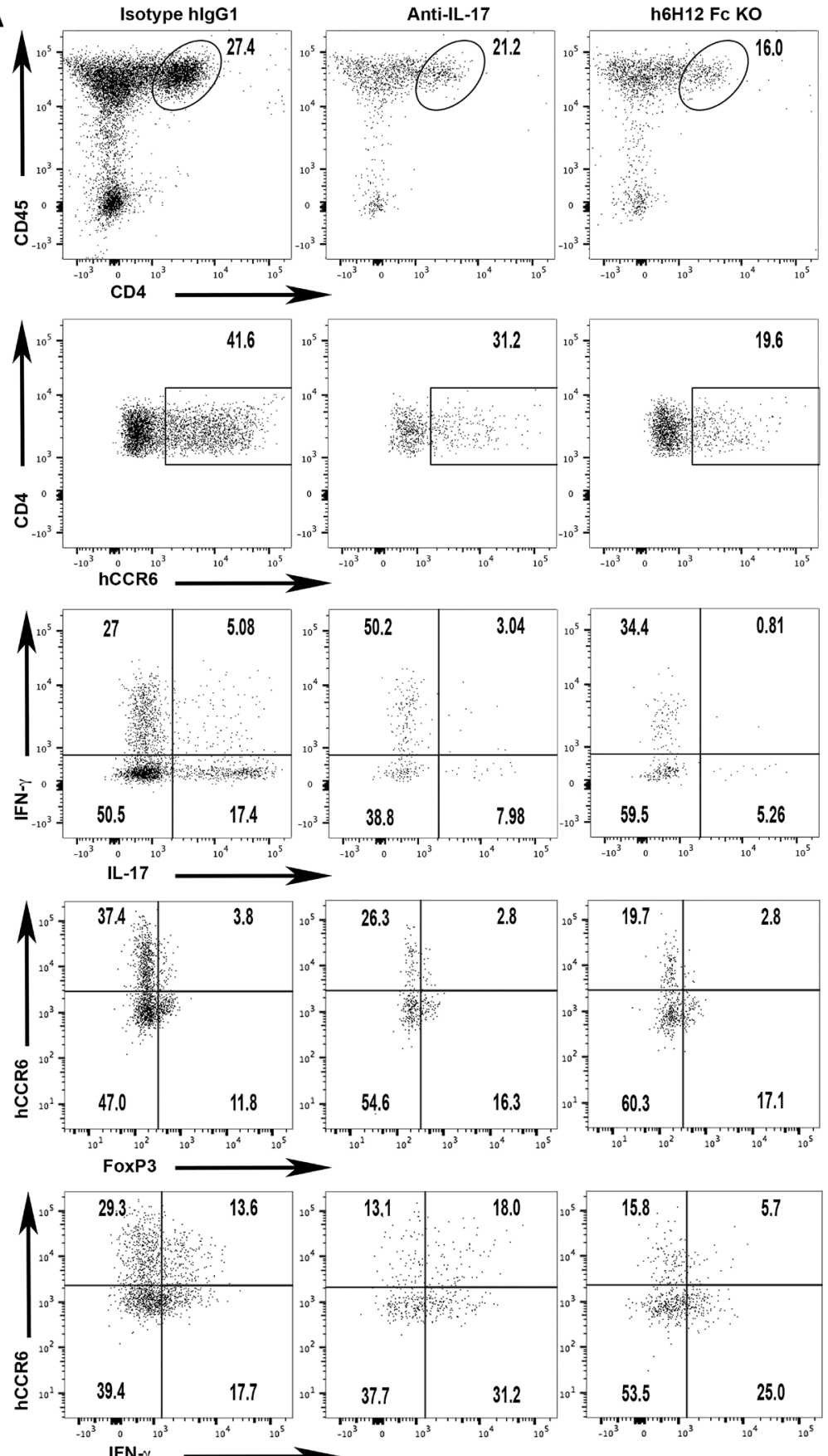

8.0

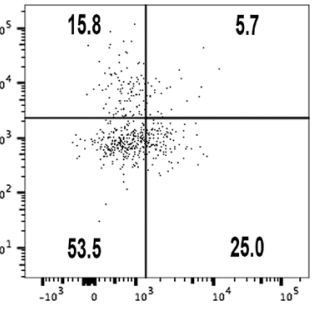

B
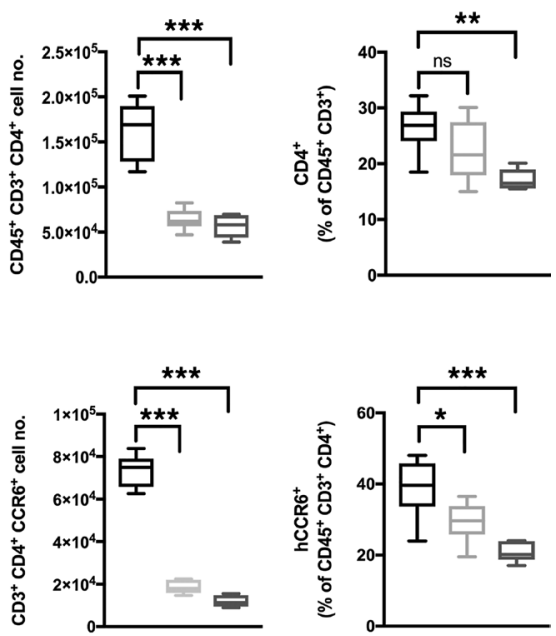
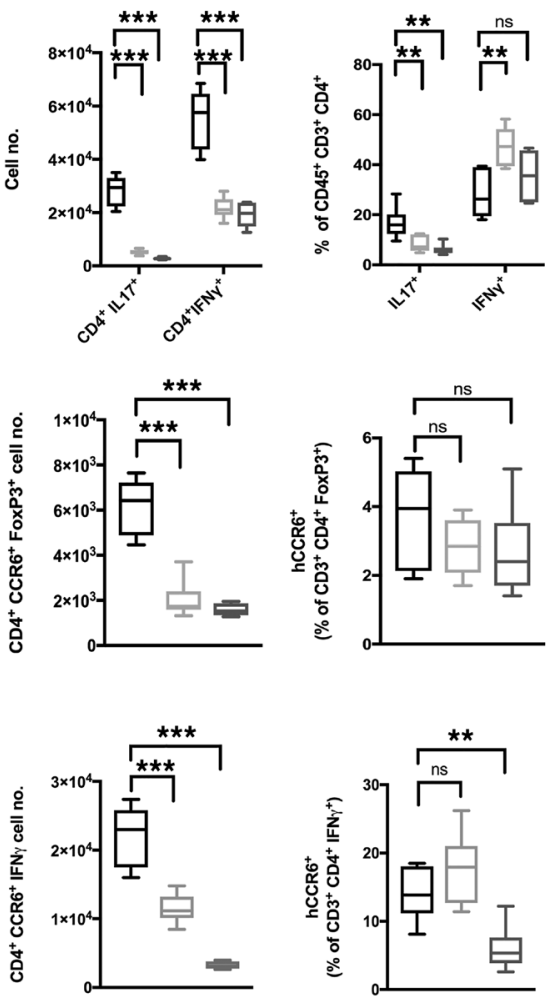

Figure 4. Anti-hCCR6 mAb prevents infiltration of leukocytes in the CNS. (A) Leukocytes were recovered from the CNS of isotype- (left panels) anti-mIL-17 mAb- (middle panels), and h6H12-treated (right panels) mice at 24 days after antigen immunization and analyzed by flow cytometry. First row, representative flow cytometry demonstrating CD45+CD4+ cells in the CNS (live cell gate). Second row, CCR6 expression of CD4+ $T$ cells (CD45+CD3 ${ }^{+}$gate). Third row, IL-17 and IFN- $\gamma$ intracellular staining of CD4+ $T$ cells isolated from CNS and stimulated for 5 hours with PMA and ionomycin. Fourth row: CCR6 and FoxP3 intracellular staining of CD4 ${ }^{+} \mathrm{T}$ cells isolated from CNS. Fifth row: CCR6 and IFN- $\gamma$ intracellular staining of CD4 ${ }^{+}$ T cells isolated from CNS and stimulated for 5 hours with PMA and ionomycin. All flow cytometric analyses were done on pooled CNS from 2 mice (6 mice per group). Results are representative of at least 2 independent experiments and the percentage of cells is shown. (B) Quantitative analysis of infiltrating T cells (first row), CCR6 ${ }^{+}$CD4 ${ }^{+}$T cells (second row), IL-17- and IFN- $\gamma$-producing CD4 ${ }^{+}$T cells (third row), CCR6 ${ }^{+}$Tregs (fourth row), and CCR6 $^{+}$ Th1 cells (fifth row) isolated from whole CNS samples showing total cell number (left columns) and percentage (right columns). Black (isotype; $n=6$ ); light gray (anti-IL-17 mAb, $n=6$ ); dark gray (h6H12 Fc-KO, $n=6$ ). Data are shown as mean $\pm \mathrm{SEM}$. For box-and-whisker plots, the whiskers represent the minimum and maximum values, the box boundaries represent the 25 th and 75 th percentiles, and the middle line is the median value. Statistical significance was determined by 1 -way ANOVA with Dunnett's multiple comparisons test relative to control isotype-treated group. ${ }^{*} P<0.05,{ }^{* *} P<$ $0.01,{ }^{* * *} P<0.001$. 
A

Vaseline control

Isotype hlgG1

Anti-IL-17

h6H12 Fc KO
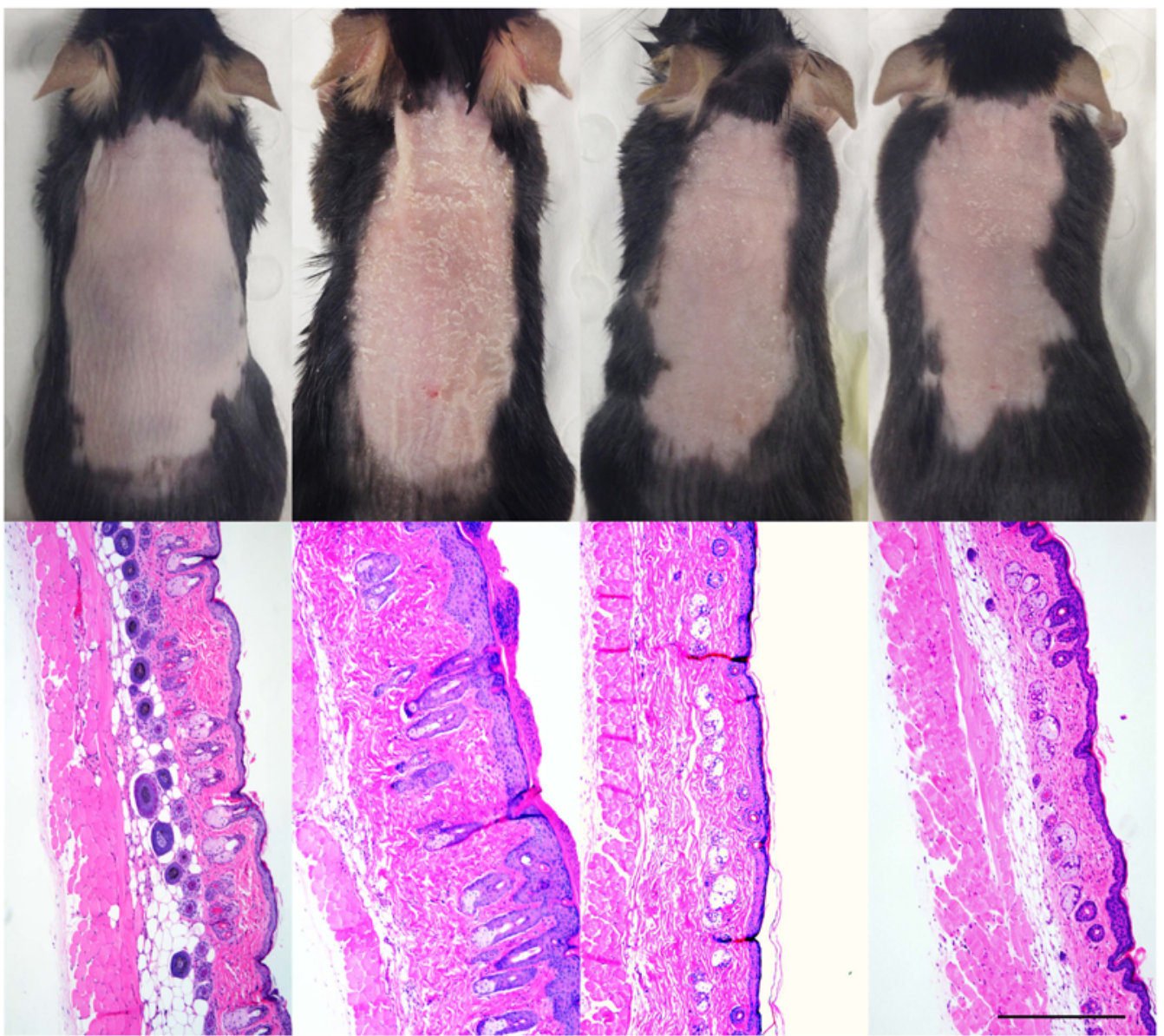

B

C
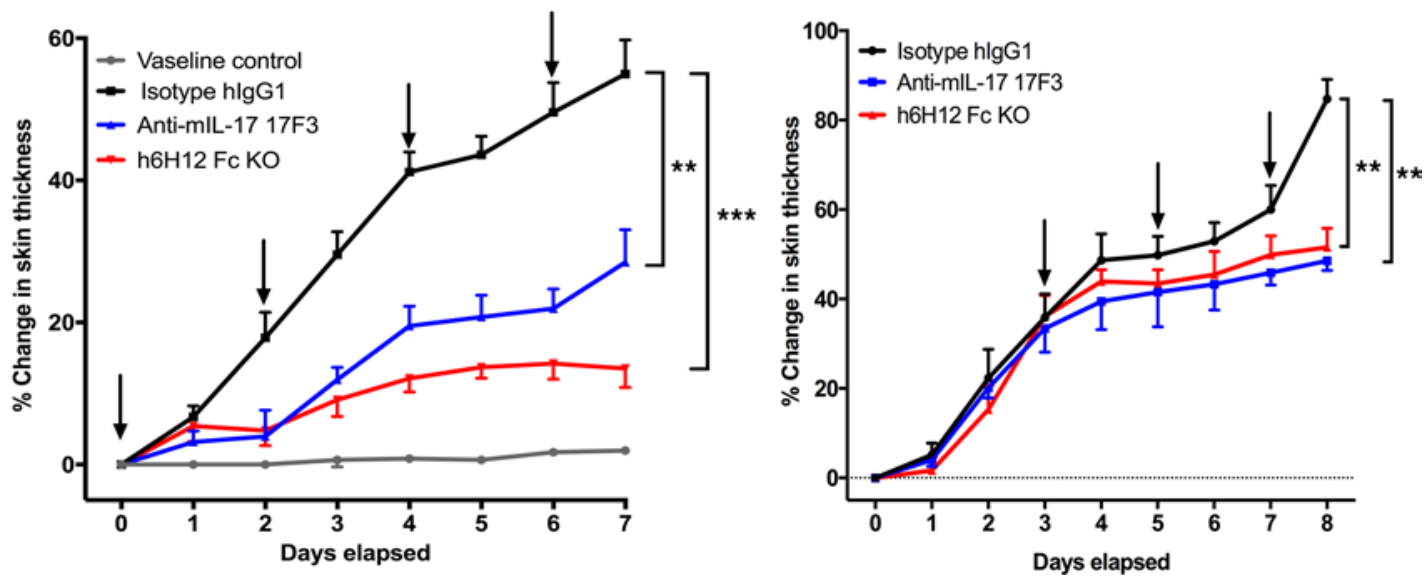

Figure 5. Anti-hCCR6 mAb prevents and inhibits imiquimod-induced psoriasis in hCCR6-Tg/mCCR6-/-mice. (A) Representative images and H\&E staining of back skin from hCCR6-Tg/mCCR6-- mice treated with Vaseline (negative control, first column); imiquimod (IMQ) + isotype control mAb (second column); IMQ + anti-mIL-17 mAb (third column); or IMQ + h6H12 Fc-KO (fourth column) ( $5 \mathrm{mg} / \mathrm{kg}$ every 2 days). (B) Dorsal skin thickness was measured daily for 7 consecutive days. Data are representative of 3 independent experiments with 6 animals per group. (C) Therapeutic study of h6H12 Fc-KO and anti-mIL-17 mAbs ( $n=10$ per group) for the treatment of IMQ-induced psoriasis. Treatment (black arrows) was started 3 days after IMQ application (20\% average change in skin thickness) and animals were monitored daily for skin thickness until day 8 . Dorsal skin thicknesses were analyzed with the nonparametric Kruskal-Wallis ANOVA with the Dunn's post-hoc test. ${ }^{* *} P<0.01,{ }^{* *} P<0.001$. 
A Control vaseline

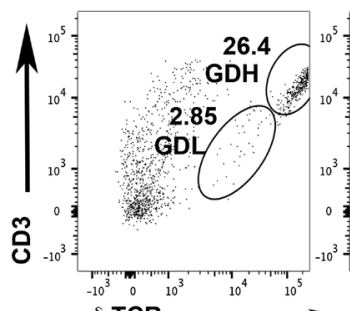

IMQ+lsotype hlgG1

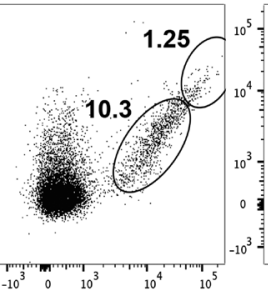

$\gamma \delta$ TCR
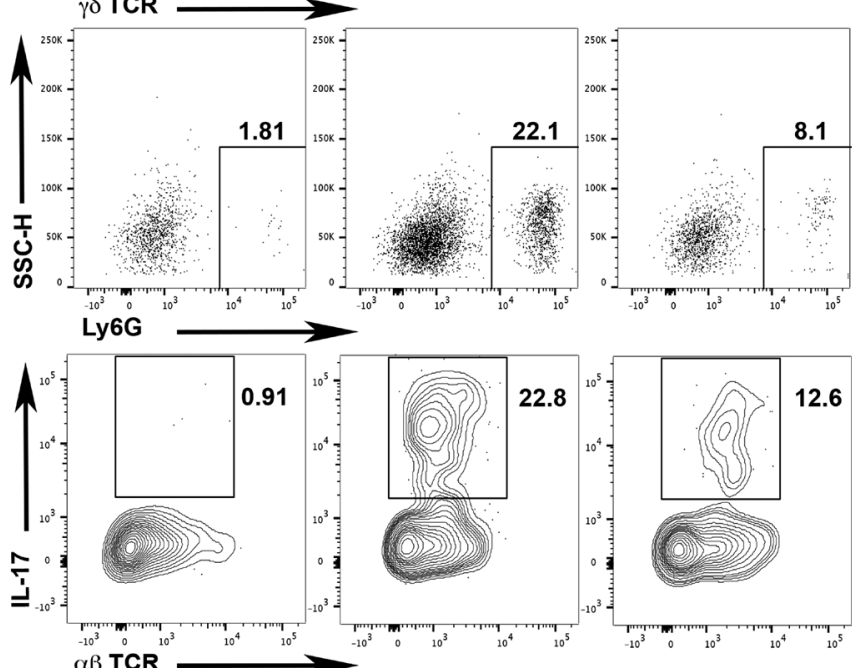

IMQ+Anti-IL-17
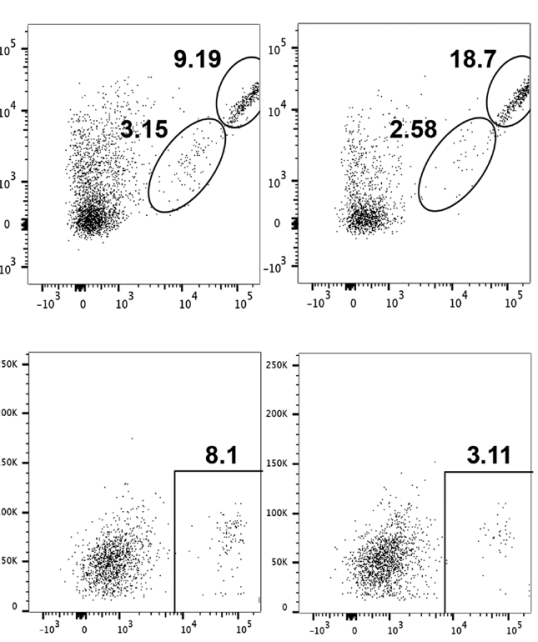

3.11

$\alpha \beta$ TCR $\longrightarrow$

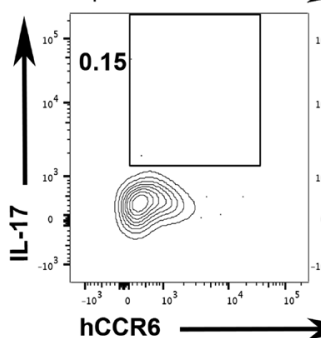

hCCR6

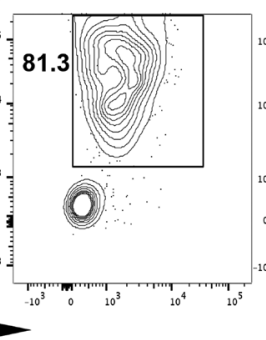

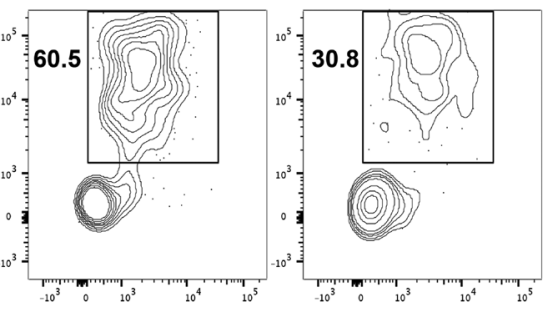
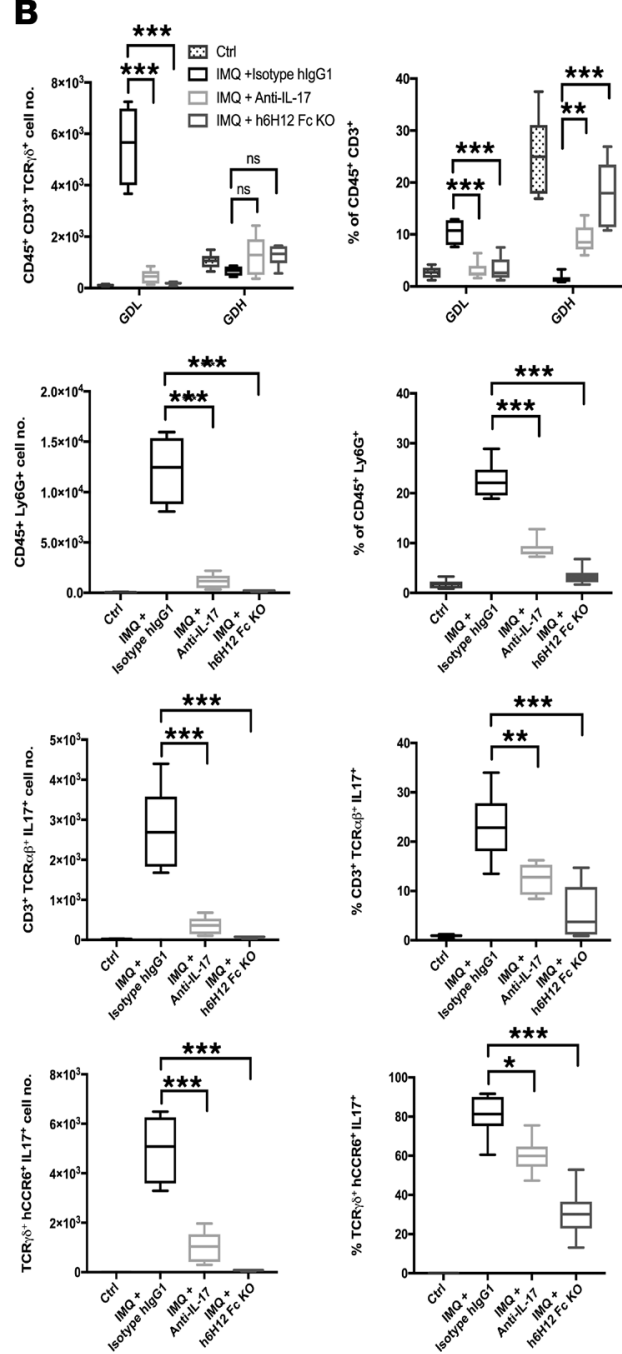

Figure 6. Anti-hCCR6 mAb prevents infiltration of leukocytes in the skin. (A) Leukocytes were recovered from the skin of mice treated with Vaseline (first column), isotype + imiquimod (IMQ) (second column), anti-mIL-17 mAb + IMQ (third column), and h6H12 + IMQ (fourth column) and analyzed by flow cytometry. All flow cytometric analyses were performed on pooled skin samples from 2 mice (6 mice per group). Results are representative of at least 2 independent experiments and the percentage of cells is shown. First row, flow cytometric analysis displaying the percentage of $\mathrm{CD}^{+} \gamma \delta \mathrm{TCR}^{\text {hi }}\left(\mathrm{GDH}\right.$, upper gate) and $\mathrm{CD}^{+} \gamma \delta \mathrm{TCR}^{10}$ (CDL, lower gate) among CD45+7-AAD- cells from skin of mice treated as described above. Second row, flow cytometric analysis showing the percentage of $\mathrm{Ly} 6 \mathrm{C}^{+}$neutrophils among CD45 7-AAD cells in the skin. Third row, IL-17 intracellular staining

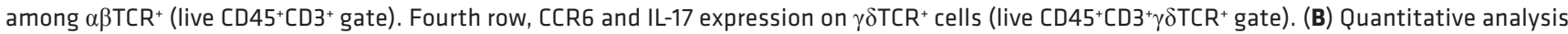
of GDH and GDL T cells (first row); neutrophils (CD45+Ly6C+, second row); IL-17-producing $\alpha \beta T C R^{+}$T cells (third row); and IL-17-producing CCR6 $\gamma \delta$ T cells (fourth row) isolated from whole $(2 \times 3 \mathrm{~cm}$ ) dorsal skin samples showing total cell number (left column) and percentage (right column). Data are shown as the mean \pm SEM, $n=6$ per group. For box-and-whisker plots, the whiskers represent the minimum and maximum values, the box boundaries represent the 25 th and 75 th percentiles, and the middle line is the median value. Statistical significance was determined by 1 -way ANOVA with Dunnett's multiple comparisons test relative to control isotype-treated group. ${ }^{*} P<0.05,{ }^{* *} P<0.01,{ }^{* *} P<0.001$.

by blocking recruitment of $\gamma \delta^{\text {lo }} \mathrm{CCR} 6^{\text {hi }} \mathrm{T}$ cells (16). Furthermore, we observed a marked reduction in neutrophil infiltration (Figure 6, A and $\mathrm{B} ; \mathrm{CD}^{+} / \mathrm{Ly}^{6} \mathrm{G}^{+}$subset), a leukocyte subset also known to contribute to chronic skin inflammation in psoriasis (33).

Intracellular cytokine staining demonstrated a marked reduction of $\mathrm{IL}-17^{+}$production by both $\alpha \beta$ $\mathrm{TCR}^{+}$and $\gamma \delta \mathrm{TCR}^{+}$CCR6-expressing subsets in the h6H12 Fc-KO- and anti-IL-17-treated groups compared with the isotype control-treated mice (Figure 6, A and B). Notably, the majority of IL-17producing $\gamma \delta \mathrm{T}$ cells appeared to be CCR $6^{+}$and anti-CCR $6 \mathrm{mAb}$ was superior to anti-IL-17 in dampening IL-17 production by both cell types. Thus, anti-CCR6 therapy could inhibit inflammatory cell infiltration into the skin, and the therapeutic benefit was superior to that observed with anti-IL-17 neutralization. 


\section{Discussion}

Until recently, chronic inflammation associated with $\mathrm{T}$ cell activation or autoreactivity was considered to derive largely from Th1 or Th2 cells. However, a major revision to this dogma occurred with the discovery of Th17 cells and their pathogenic role in conditions such as EAE (34). Subsequent findings identified Th22 (2), Th9 (35), as well as subsets of innate IL-17-producing immune cells (36) as important mediators of inflammation in a wide variety of pathologies, both in animal models and humans. The fact that all the aforementioned cells are operating through the CCR6/CCL20 axis to migrate to inflamed tissues encouraged us (and others) to investigate the potential of targeting this pathway for treatment of chronic inflammation and autoimmunity. Previous studies using either CCR6-KO mice (28) or anti-CCL20 antibodies (16) have demonstrated the benefits of interfering with this pathway in a wide range of inflammatory diseases. However, the inhibition of CCR6 per se has largely been hampered by a lack of effective antagonists for this receptor.

Chemokine receptor antagonists show considerable promise as antiinflammatory therapeutics, based partly on the large body of experimental evidence showing that chemokine receptor-deficient mice, or receptor antagonists, lead to diminished inflammation in numerous animal models. Chemoattractant receptors have, however, proved surprisingly difficult to antagonize with organic small molecules, possibly due to the complex binding of large ligands to multiple extracellular domains of these receptors (37). To date, no effective inhibitor of CCR6 has yet been developed for translation into clinical studies, and only weak partial antagonist polyclonal antibodies (23) or anti-mCCR6 mAbs (10) are currently available for studies in mouse models. We focused on the generation of a selective and potent antibody directed against hCCR6. This approach was favored over development of anti-CCL20 antibodies because high levels of chemokines secreted during inflammation might overcome mAb blockade, producing reduced in vivo efficacy as compared with CCR6 targeting. Furthermore, nonchemokine human $\beta$-defensins- 1 and -2 are reported to function as ligands for hCCR6 (38) and induce arrest of human Th17 cells on inflamed endothelium (39). Interestingly, human psoriasis is associated with increased $\beta$-defensin genomic copy number (40), suggesting that $\beta$-defensin may be operating through CCR6 in disease pathogenesis.

An important outcome of this study was the isolation of an anti-hCCR6 mAbs able to block CCL20induced cell migration. A humanization approach was designed to maximize similarity to the human germline sequence and, by engineering the Fc part of h6H12 according to Oganesyan et al. (20), we affected its capacity to bind to Fc $\gamma \mathrm{R}$ and induce ADCC. We were therefore able to solely assess the significance of antagonizing CCR6, rather than CCR6 $6^{+}$cell depletion.

For preclinical trials of therapeutic mAbs to be effective, their efficacy needs to be adequately evaluated in animal models of disease, yet in most cases, this is difficult because of lack of cross-reactivity with the orthologous (i.e., mouse) molecules (41). In this study, we generated and characterized hCCR6-Tg/mCCR6 ${ }^{-1}$ mice. The expression of the transgene on lymphocytes showed a distribution pattern consistent with endogenous mCCR6 in WT mice, although we noted some differences between mCCR6 and hCCR6 expression (e.g., overall higher expression of hCCR6 on human B cells and a higher percentage of CCR $6^{+} \mathrm{T}$ cells in humans than in WT mice). Interestingly, the expression levels and percentages of CCR6 ${ }^{+}$lymphocytes in our transgenic strain were closer to those reported in humans rather than in mice. This could be explained by the use of the endogenous hCCR6 promoter and regulatory regions during the design of the transgenic mice.

Seeking to translate in vitro potency to in vivo efficacy, we tested h6H12 Fc-KO in a number of inflammatory disease models and were able to demonstrate remarkable efficacy. In the rmMOG-EAE mouse mod$\mathrm{el}$, it prevented inflammatory cell infiltration to the CNS, as well as neurodegenerative signs that are a hallmark of the disease. In contrast, mice treated with an isotype control antibody developed severe symptoms with massive leukocyte infiltration to the CNS as well as axonal and myelin damage. More impressively, it strongly reduced disease symptoms in a preestablished disease setting. This last result was quite surprising given (a) the reported beneficial role that CCR6 may play in the later phase of EAE, by recruiting Tregs to the CNS (42), and (b) the chemokine receptor switch (from CCR6 to CCR2) observed on pathogenic Th17 cells in the later phase of EAE (43). It is important to note that this is to our knowledge the first time that CCR6 inhibition has been analyzed in this particular EAE model (rmMOG-induced model) and it cannot be ruled out that participating Tregs may be using other chemokine receptors (i.e., CXCR3 and/or CCR4) for their migration $(44,45)$. The recent demonstration that pathogenic myelin-reactive $\mathrm{T}$ cell clones $(46)$ and non-classic Th1- and GM-CSF-secreting Th cells (47) derived from human MS patients are CCR6 ${ }^{+}$supports the notion that CCR6 inhibition in MS may be equally efficacious. 
Similarly, in the IMQ-induced skin inflammation model, h6H12 Fc-KO had a striking effect in preventing epidermal hyperplasia and dermal inflammation. As previously reported by Mabuchi et al. (16), the analysis of single-cell suspensions isolated from mouse psoriatic skin revealed that the main pathogenic lymphocyte population present following IMQ application was a subset of $\gamma \delta^{\text {lo }} \mathrm{CCR} 6^{\mathrm{hi}} \mathrm{T}$ cells and that antiCCL20 mAb treatment strongly inhibited their recruitment. Importantly, analysis of skin samples derived from psoriatic patients showed increased infiltration of IL-17-producing $\gamma \delta \mathrm{T}$ cells (32). To our knowledge, this is the first time that the efficacy of an anti-CCR6 mAb has been demonstrated in a preclinical model of psoriasis. These results are in line with the observation that CCR6 or $\gamma \delta$ deficiencies (32), as well as antiCCL20 treatment (16), impair the development of psoriatic symptoms in mice.

In summary, our findings indicate that targeted inhibition of the CCR6/CCL20 proinflammatory pathway with h6H12 is a valid therapeutic approach and may be useful in the treatment of MS and psoriasis. Our results support the development of $\mathrm{h} 6 \mathrm{H} 12 \mathrm{mAb}$ therapy for these and possibly other immune-mediated disorders where $\mathrm{CCR}^{+}$cells play a pathogenic role (48). We believe that inhibition of CCR6 ${ }^{+}$cell migration would be potentially more advantageous than current anti-IL-17/IL-17R therapies, by allowing concomitant targeting of other cytokine pathways associated with CCR6 $6^{+}$cells, such as IL-22 and GMCSF, and possibly $\beta$-defensin binding.

\section{Methods}

Generation of anti-CCR6 antibodies. mAbs reactive with CCR6 were generated by immunizing C57BL/6 mice (purchased from Monash Animal Research Platform) with 107 L1.2 hCCR6-transfected cells, i.p., 5 to 6 times at 2-week intervals. The final immunization was injected i.v. Four days later, the spleen was removed and cells were fused with the SP2/0 cell line (ATCC, CRL 1581) as described previously (49).

Epitope mapping. A panel of synthetic peptides (Mimotopes) corresponding to the N-terminal region, the first, second, and third extra-cellular loops of hCCR6 (ECL1, ECL2, and ECL3) were used in a standard ELISA for rough epitope mapping of the mAbs. For fine epitope mapping, a series of 12-mer synthetic peptides (Mimotopes) with an overlap of 1 aa covering the N-terminal sequence of hCCR6 (aa 1-40) was coated directly at $1 \mu \mathrm{g} / \mathrm{ml}$ onto a 96-well plate (Nunc). Briefly, the plates were washed 3 times in PBS containing $0.05 \%$ Tween 20 (T-PBS) and blocked for 1 hour with T-PBS plus $2 \%$ milk powder. After washing 3 times with T-PBS, the plate was incubated for 1 hour with $0.1 \mu \mathrm{g}$ of purified mAbs in blocking buffer. The plate was then washed and incubated for 1 hour at room temperature with a 1:5,000 dilution of goat anti-mouse IgG peroxidase conjugate (Jackson ImmunoResearch Laboratories, catalog 115-035-008). After 3 final washes, bound IgG was detected with TMB substrate and the absorbance was read at $450 \mathrm{~nm}$.

Engineering of the $6 \mathrm{H} 12$ antibody. Total RNA, extracted from the $6 \mathrm{H} 12$ hybridoma cell lines using the TRIzol Reagent (Invitrogen), was used for cDNA synthesis. The V genes were isolated from cDNA by reverse transcription PCR using primers annealing to mouse light (mIgCk-1: 5'-CTTCCACTTGACATTGATGTCTTTG-3') and heavy constant regions (mIgG2a-1: 5'-CAGGTCAAGGTCACTGGCTCAGG-3'). The VH and VL genes were sequenced and analyzed by the IMGT databases.

Antibody humanization was performed by the CDR-grafting method as previously described (50). Briefly, IMGT/V-QUEST (51) and IMGT/Junctions (52) analysis tools were used to identify human germline genes in which sequences from the variable regions of both the heavy and light chains were closely aligned with those of murine antibody $6 \mathrm{H} 12$. Framework sequences of these selected human germline genes were used as acceptor sequences for the $6 \mathrm{H} 12 \mathrm{CDRs}$. However, murine residues were retained in the critical Vernier zone. The humanized VH and VL genes were synthesized by GENEART AG.

To produce the nondepleting (Fc-KO-L234F/L235E/P331S) humanized 6H12 IgG1 (h6H12 Fc-KO), standard mutagenesis techniques were used. The sequences were confirmed on both strands by DNA sequencing.

Expression and purification of the h6H12 IgG1 in CHO cells. Mouse VH (mVH), mouse VL (mVL), humanized VH (hVH), and humanized VL (hVL) genes were amplified by PCR and subcloned into the bicistronic pJACQ- $\mathrm{G}_{1} \mathrm{~K}_{\mathrm{a}}$ mammalian expression vector containing the human $\kappa$ and human heavy chain $\operatorname{IgG}_{1}$ constant regions. The insertion was verified by restriction digestion. Recombinant DNAs were transfected into CHO-DG44 cells with the AMAXA nucleofector device (Lonza) according to manufacturer's protocol. The presence of recombinant antibody was confirmed by Western blotting using an anti-human IgG Fcspecific HRP-conjugate antibody (Jackson ImmunoResearch Laboratories, catalog 109-35-008). The transfected $\mathrm{CHO}$ cells were grown and maintained in serum-free medium (Invitrogen) for antibody production. 
The humanized IgG1 was purified from culture supernatant by affinity chromatography on a ProSep-vA protein A column (Millipore). The bound IgG1 was eluted with $0.2 \mathrm{M}$ glycine/1 M NaCl, pH 3.0 into a neutralizing solution of $1 \mathrm{M}$ Tris, $\mathrm{pH}$ 8.0, and then buffer exchanged against PBS. Purified antibodies were endotoxin free as determined by a chromogenic LAL assay (GenScript).

Flow cytometric analysis. To assess reactivity of mAbs against transfected cells, we used indirect immunofluorescence staining and flow cytometry. Cells were washed once with PBS and resuspended in $100 \mu \mathrm{PBS}$ containing $2 \%$ BSA and $0.1 \%$ sodium azide (staining buffer) and purified antibody. After 30 minutes at $4^{\circ} \mathrm{C}$, cells were washed twice with staining buffer and resuspended in $50 \mu 1 \mathrm{PE}$-conjugated antihuman IgG (Jackson ImmunoResearch Laboratories, catalog 109-115-098) diluted 1:500 in staining buffer for the detection of humanized $\mathrm{mAbs}$ or $50 \mu \mathrm{PE}$-conjugated anti-mouse IgG (Jackson ImmunoResearch Laboratories, catalog 115-115-205) for the detection of mouse mAbs. After incubating for 20 minutes at $4^{\circ} \mathrm{C}$, cells were washed twice with staining buffer and analyzed on an LSRII flow cytometer (BD Biosciences). 7-AAD staining was used to exclude dead cells. To validate expression of chemokine receptors on transfected L1.2 cells, the following commercial antibodies were used: anti-mCCR6 (clone FAB590A, R\&D Systems, catalog FAB590A); anti-hCCR6 (clone G034E3, Biolegend, catalog 353410); anti-hCCR5 (clone 2D7, BD Biosciences, catalog 555993); anti-hCCR7 (clone G043H7, Biolegend, catalog 353218); anti-hCCR8 (clone L263G8, Biolegend, catalog 360604); anti-hCCR9 (clone MAB179, R\&D Systems, catalog MAB179F).

To assess reactivity of $\mathrm{mAbs}$ against human lymphocytes, fresh human PBMCs were prepared from heparinized blood (obtained from healthy individuals) by centrifugation on a Ficoll gradient. PMBCs were then incubated with anti-CD19 (clone HIB-19, Biolegend, catalog 302222); anti-CD3 (clone UCHT1, Biolegend, catalog 300423); anti-CD4 (clone OKT4, Biolegend, catalog 317433); anti-CD8 (clone OKT8, eBioscience, catalog 12-0086-41); and FITC-conjugated anti-hCCR6 antibodies (clone G034E3, Biolegend, clone 6H12, or clone 29A6). The cells were analyzed on an LSRII flow cytometer (BD Biosciences). 7-AAD staining was used to exclude dead cells.

Chemotaxis assay. L1.2 cells stably transfected with human CCR6 were maintained in suspension in RPMI 1640 containing 10\% heat-inactivated FCS, 2\% L-glutamine, and $100 \mathrm{U} / \mathrm{ml}$ penicillin. For the assay, the cells were washed once in PBS and resuspended at $10^{6}$ cells $/ \mathrm{ml}$ in assay buffer (RPMI 1640, $1 \%$ endotoxin-free BSA, $100 \mathrm{U} / \mathrm{ml}$ penicillin, and $100 \mu \mathrm{g} / \mathrm{ml}$ streptomycin). Antibodies were preincubated with 100 $\mu 1$ of cells $\left(1 \times 10^{5}\right.$ cells $)$ for 30 minutes and placed into the upper chamber of a transwell plate with $5-\mu \mathrm{m}$ pores (Corning Life Sciences). In the lower chambers, $120 \mathrm{nM}$ human CCL20 was added and the assay was incubated at $37^{\circ} \mathrm{C}$ for 4 hours $\left(5 \% \mathrm{CO}_{2}\right)$. Live cells migrating to the lower chamber were counted using the LSRII flow cytometer. A chemotactic index was calculated by dividing the number of migrated cells in response to a specific chemokine by that in the absence of chemokine (background).

ADCC analysis by flow cytometry. The capacity of h6H12 mAb to induce ADCC was evaluated by flow cytometry as described previously (53). Briefly, hCCR6 L1.2-expressing cells were labeled with membrane dye, $\mathrm{PKH} 26$, to allow discrimination when incubated with effector cells and antibodies. Labeled target cells were washed 3 times with culture medium and resuspended in culture medium at a concentration of $1 \times 10^{6} \mathrm{cells} / \mathrm{ml}$. Labeled target cells were dispensed in round-bottomed 96-well plates $\left(1 \times 10^{5}\right.$ in $100 \mu \mathrm{l} /$ well $)$ and preincubated with $5,1,0.5$, or $0.1 \mu \mathrm{g} / \mathrm{ml}$ of h6H12, h6H12 Fc-KO, or isotype control (humanized $8.8 \mathrm{mAb}$; gift from Anthony J. Coyle, Pfizer) at $37^{\circ} \mathrm{C}$ for 30 minutes. Human PBMCs were prepared from heparinized blood (obtained from healthy individuals) by centrifugation in a Ficoll gradient. Human NK cells were next isolated from the PBMCs using MACS CD56 microbeads and MACS positive selection columns (Miltenyi Biotec) in accordance with the manufacturer's protocol. Thereafter, $2 \times 10^{5}$ purified human NK cells (effector cells), were incubated with the target cells/ antibody mixture $(\mathrm{E} / \mathrm{T}$ ratio $=2: 1)$ at $37^{\circ} \mathrm{C}$ for 3 hours in the presence of $10 \%$ heat-inactivated serum. Treated cells were washed 3 times and resuspended in $200 \mu$ of PBS. TO-PRO 3 iodide (Thermo Fisher Scientific) was added to detect cell death. As a counting standard, $20 \mu 1 /$ well CountBright absolute counting beads (Invitrogen) was added to determine cell concentration of cell subsets. Samples were acquired on the LSRII flow cytometer.

Generating hCCR6-Tg/mCCR6 ${ }^{-1}$ mice. A 120-kb human BAC clone, RP11-318P19, carrying the gene for hCCR6 was purified, trimmed and injected at $1-2 \mathrm{ng} / \mu \mathrm{l}$ into pronuclei of C57BL/6J embryos. Four founders were identified using 4 sets of PCR to ensure the presence of the entire BAC in the Tg mice. The hCCR6 expression in those founders was next confirmed by flow cytometry on blood-derived lymphocytes. The founder showing the highest expression was selected for backcrossing to mCCR6-KO mice. 
Induction and monitoring of EAE. rmMOG was provided by C.C.A. Bernard and was synthesized, purified, and refolded as previously reported (54).

EAE was induced in 8- to 12 -week-old female hCCR6-Tg/mCCR6 ${ }^{-/-}$mice by immunization with $75 \mu \mathrm{g}$ of recombinant rmMOG emulsified in Complete Freund Adjuvant (CFA, Sigma-Aldrich) containing $4 \mathrm{mg} /$ $\mathrm{ml}$ Mycobacterium tuberculosis (Difco) subcutaneously on the inner side of both hind flanks. Mice were given $350 \mathrm{ng}$ of pertussis toxin (List Biological Laboratories) i.p. on day 0 and again 48 hours later. In the preventive EAE experiments, the mAbs 8.8 (isotype control), 17F3 (anti-mIL-17, Bio X Cell, catalog BP0173), and h6H12 were injected i.p. at $2 \mathrm{mg} / \mathrm{kg}$ on day 8 and 13 after immunization. For the therapeutic EAE experiments, the mAbs were injected on day 13 and 16. Two observers blind to the experimental procedure scored EAE daily. The severity of EAE was monitored and graded on a scale of $0-5$ : 0 , no disease; 1, limp tail; 2 , hind limb weakness; 3 , hind limb paralysis; 4, hind and forelimb paralysis; and 5, moribund or dead.

For histological assessment of CNS tissue, brains and spinal cords were removed and fixed in $10 \%$ neutral-buffered formalin, paraffin embedded, and sectioned. Sections $(5 \mu \mathrm{m})$ were prepared and stained with H\&E, luxol fast blue (LFB), and Bielschowsky silver stain (BSS) and assessed for evidence of inflammation, demyelination, and axonal damage, respectively. Semiquantitative analysis was performed blind by 2 independent observers as described previously (55).

To analyze lymphocytes infiltrated into the CNS, mice were perfused via the left atria with PBS and the brain and spinal cord removed. Total leukocytes were isolated by digestion of the brain and spinal cord homogenate with collagenase and DNAse for 30 minutes at $37^{\circ} \mathrm{C}$, followed by Percoll gradient centrifugation. The CNS homogenates were pooled for each treatment group prior to analysis. The cells were surface stained with antibodies against CD45 (clone 30F11, BD Biosciences, catalog 563410), CD4 (clone RM4-5, BD Biosciences, catalog 558107), and anti-CCR6 (clone 29A6). For intracellular cytokine staining, cells were stimulated for 5 hours with $50 \mathrm{ng} / \mathrm{ml}$ phorbol 12-myristate 13-acetate (PMA) and 500 $\mathrm{ng} / \mathrm{ml}$ ionomycin in the presence of $10 \mu \mathrm{g} / \mathrm{ml}$ brefeldin A. Next, cells were surface stained, washed, fixed, and permeabilized with Cytofix/Cytoperm buffer (BD Biosciences). Intracellular cytokines were stained with antibodies against IL-17A-PECy7 (17B7, eBioscience, catalog 25-7177-82) and IFN- $\gamma$-eF450 (clone XMG1.2, eBioscience, catalog 48-7311-82).

IMQ-induced skin inflammation protocol. Backs of 6- to 8-week-old female hCCR6-Tg/mCCR6 $6^{-1-}$ mice were shaved with an electric clipper and then treated with depilatory cream (Nair) to remove hair. Two days later, 70-75 mg IMQ cream 5\% (Aldara; 3M Pharmaceuticals) or a control cream (Vaseline; Vifor) was applied daily on the back skin for 6-7 consecutive days. In the preventive IMQ experiments, the mAbs $8.8,17 \mathrm{~F} 3$, and h6H12 were injected i.p. at $2 \mathrm{mg} / \mathrm{kg}$ every 2 days. For the therapeutic IMQ experiments, the $\mathrm{mAbs}$ were injected i.p. at $2 \mathrm{mg} / \mathrm{kg}$ every 2 days starting on day 3 .

Mice were evaluated daily by an observer blind to the experimental procedure. Back redness (erythema), presence of scales (scaling), and hardness of the skin were scored using a semiquantitative scoring system from 0 to 4 based on their external physical appearance: $0=$ no skin abnormalities, $1=$ slight, $2=$ moderate, $3=$ marked, and $4=$ severe. In addition, mice were weighed, and dorsal skin thickening was monitored by measuring double-skinfold thickness using a digital micrometer (Mitutoyo).

For histopathological analysis, skin samples were fixed in $4 \%$ formaldehyde, paraffin embedded, sectioned, and stained with H\&E.

For whole-skin leukocytes analysis, fatty tissue was removed from back skin. Precut skin was digested with Liberase TL (Roche) and DNAse (Roche) in RPMI for 1 hour at $37^{\circ} \mathrm{C}$ with agitation. Cells were filtered twice through $100-\mu \mathrm{m}$ and then $40-\mu \mathrm{m}$ nylon mesh filters before staining for flow cytometric analysis.

For surface staining, the following antibodies were used: BV650-anti-CD45 (clone 30F11); APC/Cy7anti-CD3 (clone 17A2, Biolegend, catalog 100221); AF700-anti-TCR $\beta$ (clone H57-597, Biolegend, catalog 109223); PE-anti-TCR $\gamma \delta$ (clone GL3, Biolegend, catalog 118107); and FITC-anti-hCCR6 (clone 29A6). Dead cells were excluded with Zombie Aqua dye (Biolegend).

For intracellular staining, cells were activated and surface stained as described above. Anti-IL-17A PECy7 (clone 17B7) antibody was used to detect intracellular cytokines.

Statistics. The mean daily clinical course of EAE and IMQ disease activity were analyzed with the nonparametric Kruskal-Wallis ANOVA with the Dunn's post-hoc test. Histological, maximum clinical, and cumulative EAE scores were compared by 1-way ANOVA with Dunnett's multiple comparisons test relative to the control isotype-treated group. Frequency and total cell numbers from flow cytometric analyses were compared by 1-way ANOVA with Dunnett's multiple comparisons test relative to the control 
isotype-treated group and plotted as box-and-whisker plots where the whiskers represent the minimum and maximum values, the box boundaries represent the 25 th and 75 th percentiles, and the middle line is the median value. $P$ values less than 0.05 were considered significant. GraphPad Prism version 5.01 was used for the analysis. ${ }^{*} P<0.05,{ }^{* *} P<0.01,{ }^{* * *} P<0.001$.

Study approval. All experimental procedures involving mice were carried out according to protocols approved by the Animal Ethics Committees of Monash and research was conducted in accordance with NHMRC guidelines. Buffy coats were obtained from blood of healthy donors conducted in accordance with the Declaration of Helsinki, and kindly provided by the Australian Red Cross Blood Service. Their use for this project was approved by Monash University Human Research Ethics Committee (MUHREC).

\section{Author contributions}

$\mathrm{RR}, \mathrm{CCAB}$, and CRM designed the research. RR, CA, GS, LJ, EXL, LJM, and NLP performed the experiments. RR, CA, CCAB, and CRM analyzed the data. RR and CRM wrote the manuscript.

\section{Acknowledgments}

This work was supported by Australian National Health and Medical Research Council (NHMRC, grant APP1016953) and Pfizer. The Australian Regenerative Medicine Institute is supported by grants from the State Government of Victoria and the Australian Government. The authors acknowledge FlowCore for assistance with flow cytometric analysis. We also thank Jeanette Rientjes, Arianna Nenci, and Jose Gonzalez (Monash Gene Targeting Facility) for production of the human CCR6 transgenic mice.

Address correspondence to: Charles Mackay or Remy Robert, 15 Innovation Walk, Monash University, 3800, Clayton, Australia. Phone: 61.3.99050646; Email: Charles.mackay@monash.edu (C. Mackay). Phone: 61.3.99024635; Email: remy.robert@monash.edu (R. Robert).

1. Korn T, Bettelli E, Oukka M, Kuchroo VK. IL-17 and Th17 Cells. Annu Rev Immunol. 2009;27:485-517.

2. Eyerich $\mathrm{S}$, et al. Th22 cells represent a distinct human $\mathrm{T}$ cell subset involved in epidermal immunity and remodeling. $J C l i n$ Invest. 2009;119(12):3573-3585.

3. Duhen T, Geiger R, Jarrossay D, Lanzavecchia A, Sallusto F. Production of interleukin 22 but not interleukin 17 by a subset of human skin-homing memory T cells. Nat Immunol. 2009;10(8):857-863.

4. Miossec P, Kolls JK. Targeting IL-17 and TH17 cells in chronic inflammation. Nat Rev Drug Discov. 2012;11(10):763-776.

5. Yiu ZZ, Griffiths CE. Interleukin 17-A inhibition in the treatment of psoriasis. Expert Rev Clin Immunol. 2016;12(1):1-4.

6. Mackay CR. Moving targets: cell migration inhibitors as new anti-inflammatory therapies. Nat Immunol. 2008;9(9):988-998.

7. Rot A, von Andrian UH. Chemokines in innate and adaptive host defense: basic chemokinese grammar for immune cells. Annu Rev Immunol. 2004;22:891-928.

8. Mackay CR. Chemokines: immunology's high impact factors. Nat Immunol. 2001;2(2):95-101.

9. von Andrian UH, Mackay CR. T-cell function and migration. Two sides of the same coin. N Engl J Med. 2000;343(14):1020-1034.

10. Hirota K, et al. Preferential recruitment of CCR6-expressing Th17 cells to inflamed joints via CCL20 in rheumatoid arthritis and its animal model. J Exp Med. 2007;204(12):2803-2812.

11. Manel N, Unutmaz D, Littman DR. The differentiation of human T(H)-17 cells requires transforming growth factor-beta and induction of the nuclear receptor RORgammat. Nat Immunol. 2008;9(6):641-649.

12. Lee HJ, et al. Increased expression of MIP-3alpha/CCL20 in peripheral blood mononuclear cells from patients with ulcerative colitis and its down-regulation by sulfasalazine and glucocorticoid treatment. Inflamm Bowel Dis. 2005;11(12):1070-1079.

13. Lee AY, Körner H. CCR6 and CCL20: emerging players in the pathogenesis of rheumatoid arthritis. Immunol Cell Biol. 2014;92(4):354-358.

14. Tao J, et al. Up-regulation of CC chemokine ligand 20 and its receptor CCR6 in the lesional skin of early systemic sclerosis. Eur J Dermatol. 2011;21(5):731-736.

15. Reboldi A, et al. C-C chemokine receptor 6-regulated entry of TH-17 cells into the CNS through the choroid plexus is required for the initiation of EAE. Nat Immunol. 2009;10(5):514-523.

16. Mabuchi T, et al. CCR6 is required for epidermal trafficking of $\gamma \delta$-T cells in an IL-23-induced model of psoriasiform dermatitis. J Invest Dermatol. 2013;133(1):164-171.

17. Chung SH, Chang SY, Lee HJ, Choi SH. The C-C chemokine receptor 6 (CCR6) is crucial for Th2-driven allergic conjunctivitis. Clin Immunol. 2015;161(2):110-119.

18. Bracke KR, et al. Cigarette smoke-induced pulmonary inflammation and emphysema are attenuated in CCR6-deficient mice. J Immunol. 2006;177(7):4350-4359.

19. Duhen R, Glatigny S, Arbelaez CA, Blair TC, Oukka M, Bettelli E. Cutting edge: the pathogenicity of IFN- $\gamma$-producing Th17 cells is independent of T-bet. J Immunol. 2013;190(9):4478-4482.

20. Oganesyan V, Gao C, Shirinian L, Wu H, Dall'Acqua WF. Structural characterization of a human Fc fragment engineered for lack of effector functions. Acta Crystallogr D Biol Crystallogr. 2008;64(Pt 6):700-704. 
21. Ai LS, Lee SF, Chen SS, Liao F. Molecular characterization of CCR6: involvement of multiple domains in ligand binding and receptor signaling. J Biomed Sci. 2004;11(6):818-828.

22. Varona R, et al. CCR6-deficient mice have impaired leukocyte homeostasis and altered contact hypersensitivity and delayedtype hypersensitivity responses. J Clin Invest. 2001;107(6):R37-R45.

23. Liston A, et al. Inhibition of CCR6 function reduces the severity of experimental autoimmune encephalomyelitis via effects on the priming phase of the immune response. J Immunol. 2009;182(5):3121-3130.

24. Molnarfi N, et al. MHC class II-dependent B cell APC function is required for induction of CNS autoimmunity independent of myelin-specific antibodies. J Exp Med. 2013;210(13):2921-2937.

25. Khmaladze Ia, et al. Mannan induces ROS-regulated, IL-17A-dependent psoriasis arthritis-like disease in mice. Proc Natl Acad Sci U S A. 2014;111(35):E3669-E3678

26. Lim H, et al. Proatherogenic conditions promote autoimmune T helper 17 cell responses in vivo. Immunity. 2014;40(1):153-165

27. Kulig P, et al. IL-12 protects from psoriasiform skin inflammation. Nat Commun. 2016;7:13466.

28. Hedrick MN, et al. CCR6 is required for IL-23-induced psoriasis-like inflammation in mice. J Clin Invest. 2009;119(8):2317-2329.

29. Homey B, et al. Up-regulation of macrophage inflammatory protein-3 alpha/CCL20 and CC chemokine receptor 6 in psoriasis J Immunol. 2000;164(12):6621-6632.

30. Kagami S, Rizzo HL, Lee JJ, Koguchi Y, Blauvelt A. Circulating Th17, Th22, and Th1 cells are increased in psoriasis. J Invest Dermatol. 2010;130(5):1373-1383.

31. Mabuchi T, Takekoshi T, Hwang ST. Epidermal CCR6 ${ }^{+} \gamma \delta$ T cells are major producers of IL-22 and IL-17 in a murine model of psoriasiform dermatitis. J Immunol. 2011;187(10):5026-5031.

32. Cai Y, et al. Pivotal role of dermal IL-17-producing $\gamma \delta$ T cells in skin inflammation. Immunity. 2011;35(4):596-610.

33. Sumida $\mathrm{H}$, et al. Interplay between CXCR2 and BLT1 facilitates neutrophil infiltration and resultant keratinocyte activation in a murine model of imiquimod-induced psoriasis. J Immunol. 2014;192(9):4361-4369.

34. Zhang GX, et al. Induction of experimental autoimmune encephalomyelitis in IL-12 receptor-beta 2-deficient mice: IL-12 responsiveness is not required in the pathogenesis of inflammatory demyelination in the central nervous system. J Immunol. 2003;170(4):2153-2160.

35. Dardalhon V, et al. IL-4 inhibits TGF-beta-induced Foxp3 ${ }^{+}$T cells and, together with TGF-beta, generates IL-9+ IL-10+ Foxp3(-) effector T cells. Nat Immunol. 2008;9(12):1347-1355.

36. Hirota K, Ahlfors H, Duarte JH, Stockinger B. Regulation and function of innate and adaptive interleukin-17-producing cells. EMBO Rep. 2012;13(2):113-120.

37. Klarenbeek A, et al. Targeting chemokines chemokine receptors with antibodies. Drug Discov Today Technol. 2012;9(4):e227-e314.

38. Yang D, et al. Beta-defensins: linking innate and adaptive immunity through dendritic and T cell CCR6. Science. 1999;286(5439):525-528.

39. Ghannam S, et al. CCL20 and $\beta$-defensin-2 induce arrest of human Th17 cells on inflamed endothelium in vitro under flow conditions. J Immunol. 2011;186(3):1411-1420.

40. Hollox EJ, et al. Psoriasis is associated with increased beta-defensin genomic copy number. Nat Genet. 2008;40(1):23-25.

41. Lee $\mathrm{H}$, et al. Human C5aR knock-in mice facilitate the production and assessment of anti-inflammatory monoclonal antibodies Nat Biotechnol. 2006;24(10):1279-1284.

42. Villares R, et al. CCR6 regulates EAE pathogenesis by controlling regulatory CD4+ T-cell recruitment to target tissues. Eur $J$ Immunol. 2009;39(6):1671-1681.

43. Kara EE, et al. CCR2 defines in vivo development and homing of IL-23-driven GM-CSF-producing Th17 cells. Nat Commun. 2015;6:8644.

44. O'Connor RA, Floess S, Huehn J, Jones SA, Anderton SM. Foxp3 ${ }^{+}$Treg cells in the inflamed CNS are insensitive to IL-6-driven IL-17 production. Eur J Immunol. 2012;42(5):1174-1179.

45. Lee I, Wang L, Wells AD, Dorf ME, Ozkaynak E, Hancock WW. Recruitment of Foxp3 ${ }^{+}$T regulatory cells mediating allograft tolerance depends on the CCR4 chemokine receptor. J Exp Med. 2005;201(7):1037-1044.

46. Cao Y, et al. Functional inflammatory profiles distinguish myelin-reactive T cells from patients with multiple sclerosis. Sci Transl Med. 2015;7(287):287ra74

47. Restorick SM, et al. CCR6(+) Th cells in the cerebrospinal fluid of persons with multiple sclerosis are dominated by pathogenic non-classic Th1 cells and GM-CSF-only-secreting Th cells. Brain Behav Immun. 2017;64:71-79.

48. Cao Y, Amezquita RA, Kleinstein SH, Stathopoulos P, Nowak RJ, O'Connor KC. Autoreactive T cells from patients with myasthenia gravis are characterized by elevated IL-17, IFN- $\gamma$, and GM-CSF and diminished IL-10 production. J Immunol. 2016;196(5):2075-2084

49. Wu L, et al. CCR5 levels and expression pattern correlate with infectability by macrophage-tropic HIV-1, in vitro. J Exp Med. 1997;185(9):1681-1691.

50. Robert R, Streltsov VA, Newman J, Pearce LA, Wark KL, Dolezal O. Germline humanization of a murine Abeta antibody and crystal structure of the humanized recombinant Fab fragment. Protein Sci. 2010;19(2):299-308.

51. Giudicelli V, Chaume D, Lefranc MP. IMGT/V-QUEST, an integrated software program for immunoglobulin and T cell receptor V-J and V-D-J rearrangement analysis. Nucleic Acids Res. 2004;32(Web Server issue):W435-W440.

52. Yousfi Monod M, Giudicelli V, Chaume D, Lefranc MP. IMGT/JunctionAnalysis: the first tool for the analysis of the immunoglobulin and T cell receptor complex V-J and V-D-J JUNCTIONs. Bioinformatics. 2004;20 Suppl 1:i379-i385.

53. Lee-MacAry AE, et al. Development of a novel flow cytometric cell-mediated cytotoxicity assay using the fluorophores PKH-26 and TO-PRO-3 iodide. J Immunol Methods. 2001;252(1-2):83-92.

54. Clements CS, et al. The crystal structure of myelin oligodendrocyte glycoprotein, a key autoantigen in multiple sclerosis. Proc Natl Acad Sci U S A. 2003;100(19):11059-11064.

55. Payne NL, et al. Comparative study on the therapeutic potential of neurally differentiated stem cells in a mouse model of multiple sclerosis. PLoS One. 2012;7(4):e35093. 